\title{
A vueltas con la exposición de cadáveres en Cogotas I: La evidencia del Cerro de la Cabeza (Ávila)
}

\section{Revisiting the corpses exposure in Cogotas I: The evidence of Cerro de la Cabeza (Ávila)}

JAVIER VELASCO-VÁZQUEZ

Departamento de Ciencias Históricas. Universidad de Las Palmas de Gran Canaria. C/ Pérez del Toro, 1, 35004, Las Palmas de Gran Canaria.

Email: javier.velasco@ulpgc.es

ORCID: https://orcid.org/0000-0003-0339-3500

\section{ÁNGEL ESPARZA-ARROYO}

Grupo de Investigación Reconocido PREHUSAL. Departamento de Prehistoria, $\mathrm{H}^{\mathrm{a}}$ Antigua y Arqueología. Universidad de Salamanca.

Email: esparza@usal.es

ORCID: https://orcid.org/0000-0002-9139-0774

\section{VERÓNICA ALBERTO-BARROSO}

Tibicena Arqueología y Patrimonio. Grupo de Investigación TARHA. Departamento de Ciencias Históricas. Universidad de Las Palmas de Gran Canaria.

Email: veroalberto1@gmail.com

ORCID: https://orcid.org/0000-0001-8583-5581

Recibido: 22/09/2018. Aceptado: 12/11/2018.

Cómo citar: Velasco Vázquer, Javier, Esparza Arroyo, Ángel, Alberto Barroso, Verónica (2018): “A vueltas con la exposición de cadáveres en Cogotas I: La evidencia del Cerro de la Cabeza (Ávila)”. BSAA arqueología, LXXXIV, pp. 134-167.

DOI: https://doi.org/10.24197/ba.LXXXIV.2018.134-167

Resumen: Avanzar en la explicación histórica de la diversidad y complejidad de las manifestaciones mortuorias de Cogotas I (Bronce Medio y Tardío, ca. 1800-1100 cal BC) implica necesariamente afrontar una valoración exhaustiva de cada uno de los contextos con restos humanos y, en particular, de los agentes deposicionales y posdeposicioales que participan en su definitiva configuración arqueológica. En este caso, un nuevo examen del enterramiento en hoyo del Cerro de la Cabeza (Ávila), inicialmente adscrito al Bronce Final-Hierro I, pero cuya cronología revisada lo sitúa en la plenitud de Cogotas I, ha permitido identificar las huellas de carnívoros y la acción del fuego sobre los registros esqueléticos de los dos individuos allí documentados. Estos datos, sumados al establecimiento de la secuencia y los tiempos de 
formación del depósito, aportan nueva evidencia sobre la hipótesis de la exposición de cadáveres como una práctica habitual en este contexto cronológico-cultural.

Palabras clave: Península Ibérica, Cogotas I, Bronce, tafonomía, cánidos, fuego.

Abstract: Advancing in the historical explanation of the diversity and complexity of the mortuary manifestations of Cogotas I (Iberian Bronze Age; MBA and LBA, ca. 1800-1100 cal BC) implies that, necessarily, an exhaustive evaluation of each of the contexts with human remains must be faced, and, in particular, of the depositional and postdepositional agents involved in their definitive archaeological configuration. This paper addresses a review of El Cerro de la Cabeza burial pit (prov. Ávila), initially ascribed to the LBA-EIA period, but that the new dates assign to Cogotas I. The results have allow to identify some evidences related to the activity of carnivores and to the action of fire in the skeletal remains of the two individuals documented there. These data, added to the establishment of the sequence and the times relating to the formation of the funeral deposit, provide new evidence on the hypothesis of the exposure of corpses as a common practice for this chrono-cultural context.

Keywords: Iberian Peninsula, Cogotas I, Bronze Age, taphonomy, canids, fire.

\section{INTRODUCCIÓN}

El análisis tafonómico de los contextos arqueológicos con restos humanos constituye una de las estrategias fundamentales desde las que abordar cualquier comportamiento del pasado en el que esos materiales hayan tenido algún tipo de protagonismo. Desde esta premisa, y con el ánimo de explicar las particularidades del registro mortuorio en Cogotas I, se ha propuesto la hipótesis de la exposición de los cadáveres como ritual normativo (Esparza et alii, 2012b), o, mejor, como la práctica más extendida en esta población (Boulestin, 2016: 376). Así, en probable relación con una ideología dualista, de buena y mala muerte (vid. Thomas, 1982: 101ss, Insoll, 2015: 155), los fallecidos de manera considerada "natural" serían sometidos a exposición; en cambio, los muertos en circunstancias anómalas —por ejemplo, como consecuencia de accidentes, epidemias, etc. - o mal considerados socialmente quedarían fuera de la norma habitual, siendo enterrados en el interior de los poblados, en hoyos, ya fuesen construidos ad hoc o, como parece predominante, silos agotados.

Esa hipótesis de trabajo constituye apenas un primer paso que requiere, no solo del estudio de nuevos enclaves en los que se atestigüe ese comportamiento, sino también ofrecer explicaciones de alcance más global y que atiendan a la casuística tan compleja observada hasta el momento en este contexto cronológico. En este sentido es imprescindible analizar en detalle la variabilidad detectada en los enterramientos en hoyo, que incluyen depósitos secundarios y primarios, así como, entre ellos, un porcentaje destacado de múltiples. 
Enterramientos en los que, además, se observa una materialización arqueológica tan diversa como el de la mujer con su feto a término de Los Tolmos (Esparza et alii, 2017), el particular ejemplo de los subadultos de La Horra (Palomino et alii, 1999; Esparza et alii 2012a: 307) o de Castellanos de Villiquera (Alacet, 2008), o los cuatro individuos a los que se dio muerte violenta y sepultura simultánea en Los Rompizales (Velasco y Esparza, 2016).

Como ya se adelantaba, es también imprescindible valorar nuevos ejemplos de exposición, ya que la propuesta inicial se basaba esencialmente en dos casos del mismo yacimiento en los que se documentaron huellas evidentes dejadas por mordeduras de cánidos y una prolongada exposición subaérea. Un primer paso en esta dirección es recopilar las contadas referencias a casos de exposición publicados en la Submeseta Sur, donde ya se había sugerido que "hasta que no dispongamos de nuevos datos tenemos que pensar que, o bien no estaba generalizada la práctica de las inhumaciones, dejándose los cuerpos a la intemperie, o incinerándose sin dejar ningún rastro. Concretamente para la primera de esas dos hipótesis contamos, en el Horizonte Cogotas I, con la aparición de restos humanos muy fragmentados asociados a desechos de cocina" (Blasco et alii, 1991: 69). Paulatinamente se han ido publicando algunos ejemplos madrileños, como el del fondo 128 de la Fábrica de Ladrillos (Getafe), en el que se señala la presencia de un húmero cuyo distal ha sido destruido por mordeduras, alteraciones que afectan también a la fauna del relleno (Blasco et alii, 2007: 64). Otros hallazgos recientes son recogidos por Pérez Villa (2015), como el depósito primario de un individuo infantil inhumado en el hoyo UE 6010, seguramente cogotense, de Soto de Henares, que presentaba "marcas de dientes en el húmero izquierdo, posiblemente producidas por algún animal pequeño” (ibid.: 236). Más interesantes, por la seguridad de su datación en la fase ProtoCogotas I, son los dos casos del yacimiento ERAR Butarque: en primer lugar, un individuo inhumado en el hoyo K-XVI en el que "se comprobó la presencia de marcas de roedores en los huesos largos, no pudiendo precisar si se produjeron dentro de la sepultura, o por el contrario, debido a la exposición del cadáver a la intemperie” (ibid.: 205). En segundo lugar, el único resto humano identificado en el hoyo K-XXIV, una porción proximal de fémur, muestra una serie de marcas que pueden ser atribuibles a mordeduras de carnívoro (ibid.: lám. 37), con destrucción de parte de la epífisis y la presencia de surcos e impactos de caninos en la cabeza.

Por su parte, se reinterpreta como caso de exposición un viejo hallazgo, el del individuo desmembrado y guardado en vasijas del fondo 20 del Caserío de Perales, (Blasco et alii, 1991), que según Aliaga y Megías (2011: 182) sería un "...cadáver descuartizado que podría haberse obtenido de forma pasiva, ya que no existen marcas de cortes en los huesos, mediante la exposición del cuerpo a la intemperie. A favor de la existencia de rituales de exposición y enterramientos secundarios como posible explicación a estos hallazgos, habría 
que añadir que esta hipótesis permitiría explicar también la presencia de los restos óseos aislados aparecidos dentro de los hoyos basureros antes mencionados, que serían el resultado de la limpieza de las áreas de exposición tras la recogida de partes del cuerpo previamente seleccionadas”.

Ciertamente, los indicios se multiplican, pero el avance exige ampliar el 'cuerpo de evidencia' mediante el estudio exhaustivo de cuantos restos humanos pudieran suministrar información, a favor o en contra, sobre la hipótesis en discusión. En esta línea se presenta la revisión del hoyo del Cerro de la Cabeza (Ávila) en el que se recuperaron los restos de dos individuos, en cuyos esqueletos se observan indicios que permiten sostener que no fueron enterrados hasta un momento avanzado de su esqueletización, proceso durante el cual fueron accesibles a la acción de carroñeros (Fig. 1).

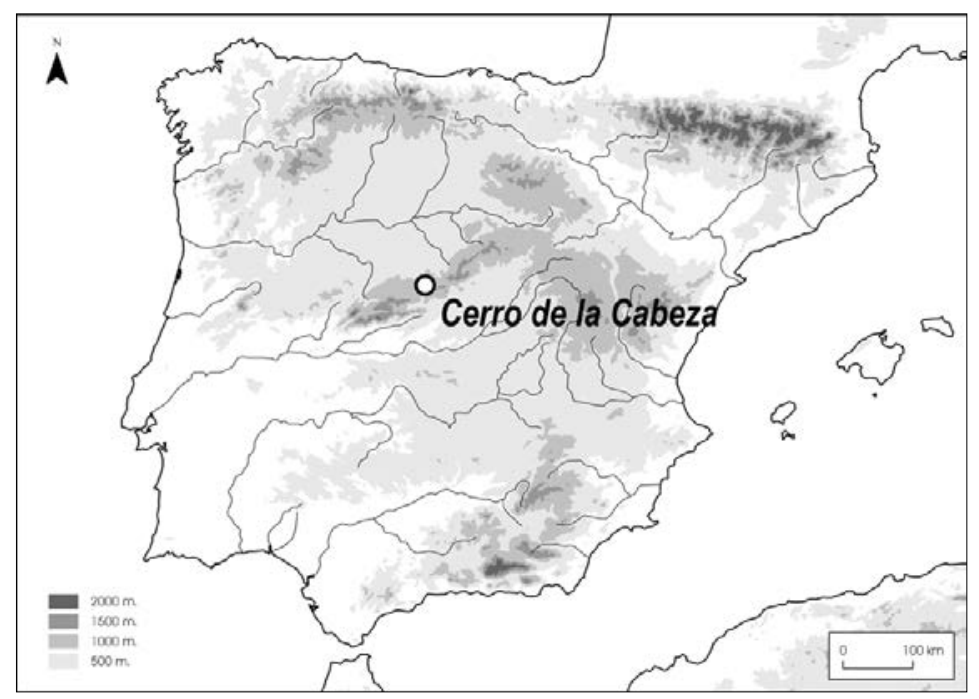

Fig. 1. Localización del yacimiento de Cerro de la Cabeza (Ávila)

\section{EL ENTERRAMIENTO Y SU ENCUADRE CRONOLÓGICO Y SOCIAL}

Este depósito de una mujer y un subadulto ya ha sido objeto de una exhaustiva publicación (Fabián et alii, 2010), a la que remitimos para los detalles generales. Fue hallado en 1997 en el Cerro de la Cabeza, a las afueras de Ávila, durante las excavaciones previas al trazado de la circunvalación de la capital, en un yacimiento con vestigios que se escalonan entre el Neolítico y la Edad del Hierro, y en el que también se documentaron cuatro enterramientos de 
finales del Calcolítico. El ejemplo que ahora nos ocupa parecería quedar encuadrado en la Segunda Edad del Hierro gracias a la datación radiocarbónica convencional de una muestra del individuo femenino (Beta-109832): $2160 \pm 50$ BP; 363-88 cal BC (92.4\%) y 77-57 cal BC (3\%). Esa datación, sin embargo, resultaba discordante con los materiales asociados a los restos humanos: dos pulseras broncíneas lisas cuya aleación rica en plomo apuntaría a un momento avanzado, incluso final, de la Edad del Bronce o comienzos del Hierro I, pero sobre todo respecto al que con mayor seguridad parece ser el elemento más reciente del relleno del hoyo, un fragmento cerámico con decoración de Boquique, asignable a la Plenitud de Cogotas I.

Ante tal problemática se procedió a la extracción de una muestra de cada uno de los individuos - fémur en el caso de la mujer (ACC01) y peroné en el subadulto (ACC02)—, cuyo análisis radiocarbónico mediante Espectrometría de Masas con Aceleradores proporcionó los siguientes resultados:

TABla I. Dataciones radiocarbónicas de los dos inhumados en el Cerro de la Cabeza (Ávila). Laboratorio de AMS de Poznan (Polonia)

\begin{tabular}{|l|l|l|l|l|}
\hline Sigla & $\begin{array}{l}\text { Refa. } \\
\text { Laboratorio }\end{array}$ & $\begin{array}{l}\text { Fecha } \\
\text { convencional }\end{array}$ & $\begin{array}{l}\text { Intervalos de calibración a 2 } \sigma \text { con } \\
\text { OxCal 4.3 (Bronk Ramsey 2017) }\end{array}$ & $\begin{array}{l}\text { Intervalos a 2 } \sigma \text { tras tomar en } \\
\text { cuenta la posición estratigráfica }\end{array}$ \\
\hline ACC-01 & Poz-49973 & $\mathbf{3 0 5 0} \pm \mathbf{3 5} \mathbf{~ B P}$ & $\mathbf{1 4 0 9}$ cal BC (95.4\%) 1219 cal BC & $\mathbf{1 4 0 9}$ cal BC (95.4\%) 1234 cal BC \\
\hline ACC-02 & Poz-49974 & $\mathbf{3 0 9 5} \pm \mathbf{3 0} \mathbf{~ B P}$ & $\mathbf{1 4 2 9}$ cal BC (95.4\%) $\mathbf{1 2 8 0}$ cal BC & $\mathbf{1 4 2 3}$ cal BC (95.4\%) 1278 cal BC \\
\hline
\end{tabular}

Las dataciones, sobre todo, vienen a despejar las dudas sobre la pertenencia del conjunto a la fase de Plenitud de Cogotas I, atribución que ya parecía desprenderse del citado fragmento cerámico. Además, a simple vista parecen concordar con el orden en que se habrían depositado los restos de los dos individuos.

Pero, dado el carácter probabilístico de las dataciones, no se resuelven todos los problemas. Así, el modelado bayesiano, también con OxCal 4.3, permite procesar junto con las dataciones el orden en que fueron depuestos los individuos, primero el subadulto y luego la mujer —implícitamente es el orden, razonablemente supuesto, de ambos fallecimientos-, pero los nuevos intervalos obtenidos mediante esa restricción, aunque más cortos, siguen siendo coincidentes en gran medida, por lo que no se puede establecer de manera concluyente ese orden ni la posible separación temporal entre los dos eventos. Ciertamente, también puede estimarse - ahora con el programa BCal (University of Sheffield, https://bcal.shef.ac.uk/) — la probabilidad de que el evento de la muerte del subadulto sea efectivamente anterior al de la mujer, obteniéndose un valor muy alto $(\mathrm{P}=0.9919607)$, pero lo verdaderamente 
relevante, la separación en el tiempo, solo se puede afrontar, como luego se verá, atendiendo a la información bioarqueológica.

En segundo lugar, estas nuevas dataciones radiocarbónicas obligan también a retomar brevemente la cronología de las pulseras que, como se ha mencionado, fueron llevadas por el alto contenido en plomo (tabla II) al Bronce Final IIIb (Fabián et alii, 2010:187) y a la transición Bronce Final / Hierro I (ibid.:188), algo alejada de la plenitud de Cogotas I.

TABLA II. Análisis compositivo de las dos pulseras realizado por S. Rovira (según Fabián et alii, 2010: 186)

\begin{tabular}{|c|l|l|l|l|l|l|l|l|l|l|l|}
\hline N $^{\circ}$ Análisis & $\mathrm{Cu}$ & $\mathrm{Sn}$ & $\mathrm{Pb}$ & $\mathrm{As}$ & $\mathrm{Fe}$ & $\mathrm{Ni}$ & $\mathrm{Zn}$ & $\mathrm{Ag}$ & $\mathrm{Sb}$ & $\mathrm{Au}$ & $\mathrm{Bi}$ \\
\hline CC1 & 86.3 & 10.3 & 3.2 & nd & 0.12 & nd & nd & 0.007 & 0.052 & - & - \\
\hline CC2 & 86.4 & 10.1 & 3.3 & nd & 0.10 & nd & nd & 0.010 & 0.047 & - & - \\
\hline
\end{tabular}

Ciertamente, no sería difícil poner el acento, más que en lo cronológico, en el tipo de objetos, pues en este caso, al tratarse de objetos de adorno y no de armas o útiles necesitados de gran dureza, la adición del plomo — que aumenta la fluidez de las coladas (Scott, 1991) - bien pudiera justificarse por la facilidad de fabricación. Pero sobre todo, deberían tenerse en cuenta los trabajos más recientes sobre la metalurgia de la zona, como el propiciado por el redescubrimiento del depósito leonés de Valdevimbre (Celis et alii, 2007a), del que se desprende una visión mucho más matizada del problema de la 'plumbificación' del Bronce Final: La complejidad de este fenómeno no permite resolverlo recurriendo, como se venía haciendo, ni a una menor disponibilidad de los otros metales necesarios, $\mathrm{Cu}$ o $\mathrm{Sn}$, ni a razones mecánicas ni a particularismos mineralógicos (vid. Delibes et alii, 2007: 118). Y desde luego, si la generalización de las aleaciones ricas en plomo se producirá en el Bronce Final III y en relación con el Soto Formativo, la presencia del plomo era ya sistemática en los fabricados metálicos de la Plenitud de Cogotas I (FernándezManzano et alii, 2005: 148; Delibes et alii, 2007: 117 y 119). No estaría de más recordar que en el enterramiento triple de San Román de la Hornija, paradigmático de esa época, había tres objetos de bronce (Delibes, 1978: 236) en cuyas aleaciones está presente el plomo: la fíbula de codo, con un 0,15\% (Herrán, 2008: 159); el pendiente, con un 0,58\% (Rovira et alii, 2004: 235), y el lingotillo que inicialmente parecía de plata pero cuyo análisis terminaría revelando un 9,106\% de plomo (Delibes y Val, 2005-2006: 305). Pero sobre todo los datos de los objetos hallados en contextos cogotenses, como los de Ardón, cuyo contenido medio en plomo es del $1,5 \%$, casi coincidente con el 
1,53\% del depósito de Valdevimbre; o los de Carricastro, con el 0,98\%, y otros, que permiten afirmar que del medio centenar de elementos metálicos de Cogotas I, un tercio contienen plomo, y en unas cantidades cuyo promedio se sitúa en el 1,3\% (Delibes et alii, 2007: 116-7; Herrán, 2008: 353; FernándezManzano y Herrán, 2012: 44 y 55). Y si hasta ahora las referencias se han ceñido a la Submeseta Norte, no es menos cierto que en los yacimientos cogotenses del valle del Tajo también se ha detectado un proceso similar (Blasco y Rovira, 1992-3): sirvan como muestra un alfiler y un arete del Caserío de Perales (Getafe, Madrid), con porcentajes de plomo del 3,4 y 7,7 respectivamente (ibid: 403). En definitiva, las pulseras que portaba el individuo femenino del Cerro de la Cabeza no serían por su composición un elemento chocante o anómalo en ese contexto de la Plenitud Cogotas I en el que el fragmento cerámico y las dataciones radiométricas sitúan el enterramiento.

Cosa bien distinta es la anomalía que desde el punto de vista social supone un ajuar, especialmente metálico, no tanto en la vida de las comunidades de Cogotas I, sino en su vertiente fúnebre. Porque, en efecto, aunque en el sector duriense de esta cultura se han hallado tres docenas de brazaletes (Herrán, 2008: 95-8) tipológicamente asignables con mucha probabilidad a esa época, la gran mayoría han salido en depósitos o fuera de contexto, aunque también hay algunas en ambientes que parecen con bastante seguridad cogotenses, pero no enterramientos, caso de los ejemplares de Ardón y El Berrueco. Tales piezas se convertirían, junto a las escasas joyas áureas, fíbulas y espadas de bronce, en argumentos en favor de la existencia de algunas personas de estatus ascendente (vid. Celis et alii, 2007b)

A este respecto, en la primera publicación, Fabián et alii (2010: 191) se alineaban con la interpretación propuesta para el caso asturiano de Fuentenegroso, donde las dos pulseras que portaba la mujer allí enterrada serían, más que un ajuar funerario, los objetos personales que "no se separan de la fallecida al ser enterrada" (Barroso et alii, 2007: 25), llegándose a sugerir que, en un contexto como el abulense, donde hubo probablemente actividad de producción metalúrgica, el (supuestamente) frecuente disfrute de objetos de bronce justificaría su amortización en el momento de morir una persona.

Sin descartar esta interpretación, que necesitaría más respaldo, debe traerse a colación el otro enterramiento de Cogotas I en el que excepcionalmente aparecen elementos de acompañamiento broncíneos, el de La Requejada (San Román de Hornija). Aquí —además de la fíbula de codo, depuesta por encima de la capa de piedras que recubría la triple inhumación y que según Delibes (1978: 229) pudiera haber sido una ofrenda colectiva, hecha para los tres difuntos, tal vez prendida sobre una capa o manto extendido en la tumba (Almagro-Gorbea, 1986: 369) - contamos con otros dos objetos metálicos, también de bronce plomoso, como vimos: el lingotillo que 
acompañaba a la mujer enterrada en el lado septentrional y el arete de bronce, un pendiente quizás, hallado junto al subadulto del lado meridional. En el conjunto de la interpretación que ha sido formulada según la cual el rito normativo en Cogotas I debió de ser la exposición de cadáveres mientras que los enterramientos en hoyo se deberían a razones muy concretas elaboradas ideológicamente como 'mala muerte', estos dos casos resultan excepcionales ya que todos los demás carecen de ajuar, por sencillo que fuese.

A decir verdad, es casi inevitable suponer la pertenencia a un sector social destacado ${ }^{1}$ para estos individuos que, aun habiendo sido víctimas de la desgraciada forma de muerte que los condujo al enterramiento en hoyo, llevaban objetos metálicos de adorno personal. Pero ¿por qué, entonces, esas personas supuestamente de rango elevado no fueron despojadas de sus elementos metálicos, como venía siendo la norma cogotense para las víctimas de 'mala muerte'? Aquí podría ser clave el detalle de la cronología de los dos enterramientos que nos ocupan, pertenecientes ambos a la Plenitud de Cogotas I: a diferencia del ProtoCogotas I, ahora, en la fase más reciente se debieron de empezar a producir brotes de diferenciación social, relajándose las normas "igualitarias", como la de la privación de objetos de acompañamiento, que hasta entonces regían el mundo de la muerte, al menos el de la 'mala muerte'. Dicho de otra forma, los mecanismos ideológicos que venían sirviendo como freno a la aparición de jerarquías, empezaban a debilitarse.

\section{EL DEPÓSITO DE RESTOS HUMANOS}

Como se ha indicado, el yacimiento fue objeto de una exhaustiva descripción arqueológica (Fabián et alii, 2010), por lo que ahora se evitará en lo posible reiterar aspectos abordados en aquel trabajo. El contenedor mortuorio en cuestión era una fosa de planta casi circular de 1,41 m de diámetro máximo y algo más de 1,00 m de profundidad en la que fueron incluidos dos individuos:

1 Frente a los argumentos ya mencionados de Celis et alii (2007b) o los de quienes propugnan la existencia de aristocracias consolidadas en Cogotas I (p.ej., Álvarez-Sanchís, 1999: 54ss), otros autores rechazan la existencia de diferencias sociales en esta cultura cuya "economía y sociedad con una organización muy elemental” no podrían haber dado lugar a la aparición de élites locales (Fernández Castro 1997: 138-140); o afirman que se trataba de grupos segmentarios relativamente igualitarios, alejados de vínculos jerárquicos (Arnáiz y Montero, 2003-4); o, admiten, como mucho, "una jerarquización que no supera un liderazgo de bajo nivel" (Fernández-Posse, 1998: 243). Más recientemente, desde una perspectiva integradora de toda la información, Blanco González (2010: 116-117) concluía que los escasos objetos suntuarios son "muy significativos en unos ambientes de absoluta invisibilidad del poder, en una cotidianeidad rústica, anodina y casi miserable", como posibles indicadores del "surgimiento de varones prominentes" y, en definitiva, de la aparición de "fisuras en el estricto ordenamiento social y productivo cogotense" (ibid.: 118). 
un subadulto, muy posiblemente varón, fallecido en torno a los 11-13 años y una mujer de aproximadamente 20-25 años (Fig. 2). En su momento, se consideró la deposición diferida de estas dos personas, donde la introducción de la segunda altera la primera y, ante la ausencia de algunos restos óseos, la posibilidad de que pudieran proceder de un enclave mortuorio diferente a la fosa en la que fueron hallados. No obstante, la revisión tafonómica que se propone para este caso revela una situación algo diferente.

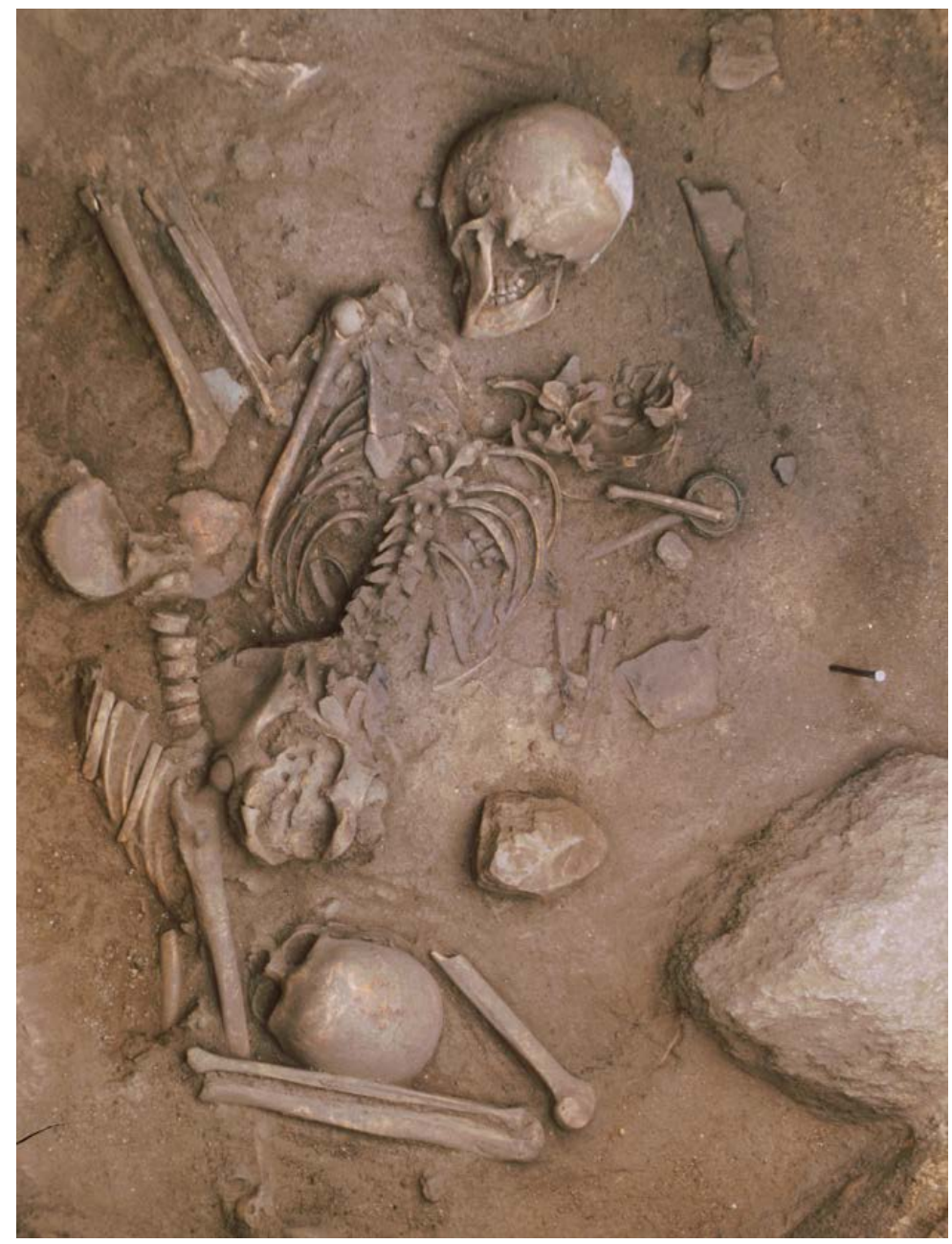

Fig. 2. Depósito mortuorio del Cerro de la Cabeza en el curso de la intervención arqueológica.

BSAA arqueología, LXXXIV, 2018, pp. 134-167.

E-ISSN: 2530-6367 
Todos los datos disponibles permiten defender que la deposición no fue completamente simultánea, en el sentido de un único evento, aún asumiendo que los tiempos funerarios entre ambas deposiciones no debieron de estar muy distanciados entre sí. De tal modo, el primero en ser introducido en la fosa fue el subadulto, dispuesto en decúbito supino con al menos la pierna derecha completamente flexionada, en una posición en apariencia forzada. De la extremidad superior, solo conservaba el brazo derecho que se encontraba ligeramente replegado. En la publicación previa se hacía constar el desplazamiento de ciertos huesos de este individuo (vértebras, costillas, etc.), en algún caso a considerable distancia de lo que debió de ser su posición primaria. Muestra de ello es la referencia para la mandíbula "situada al lado del cráneo del otro individuo, es decir a sus propios pies" (ibid.: 185). Tales movimientos fueron interpretados como el resultado de las modificaciones debidas a la introducción en el hoyo del segundo sujeto en un momento posterior. En segundo lugar fue colocada la mujer adulta prácticamente sobre el lado derecho del subadulto, en contacto directo con su cuerpo a juzgar por la información gráfica disponible, y con una orientación contraria. Su esqueleto aparece en posición de decúbito prono, con el brazo izquierdo flexionado bajo el cuerpo y el derecho, también flexionado, en paralelo a él. La pierna izquierda, solo representada por el fémur de este lado, se orienta hacia medial, mientras que en la derecha el fémur y la tibia definen un ángulo de unos $40^{\circ}$ para apoyarse sobre la anterior.

Los cuerpos, muy probablemente, llegaron al hoyo poco después de producirse la muerte y no fueron cubiertos de tierra, como así lo mostrarían los movimientos observados en ciertas regiones anatómicas de ambos sujetos. Siguiendo lo planteado en su momento, todo hace pensar que a lo largo de buena parte de la descomposición de los cadáveres, la fosa se encontrase sin colmatar, protegida quizás con algún tipo de cubierta ligera en la zona de la boca (ibid.: 188). Por los datos recabados, no parece apropiado tipificarlo como un depósito doble, al menos en lo que se refiere a la simultaneidad en la introducción de los cuerpos, sino que entre uno y otro media un tiempo difícil de precisar, pero lo suficientemente largo para que ya hubiera avanzado el proceso de esqueletización del primero en ocupar el hoyo. Así, los restos de la mujer se sitúan en contacto directo sobre los del subadulto, testimoniando la ausencia de una cobertera sedimentaria recubriendo a la primera deposición cuando se produce la segunda. En todo caso es necesario exponer bajo qué criterios se sustenta la viabilidad de esta propuesta. 


\subsection{El análisis tafonómico del depósito del Cerro de la Cabeza}

Uno de los aspectos más singulares de cuantos definen este depósito es que ambos individuos presentan una evidente subrepresentación anatómica, lo que no deja de llamar la atención en un contexto aparentemente primario donde los materiales esqueléticos presentan un óptimo estado de conservación. En la descripción original se planteó como hipótesis que tal circunstancia resultaría de una exposición previa de los cuerpos en otro lugar, donde habrían sido dañados por la acción de carroñeros, quizás aves (ibid.: 188-189). De ser así, el hoyo del Cerro de la Cabeza sería un buen ejemplo de depósito en dos tiempos (Duday et alii, 2009), lo cual, a la vista de las nuevas dataciones, casaría bien con la reciente propuesta de exposición de cadáveres como gesto funerario normativo de Cogotas I (Esparza et alii, 2012b). No obstante, ese encaje requiere de un análisis profundo que integre, más allá de la referida ausencia, otras variables que también acontecen en el hoyo.

La revisión de los materiales esqueléticos confirmó la mencionada subrepresentación esquelética, tal y como se aprecia en los esquemas que acompañan al texto. Para tratar de profundizar en esta cuestión se procedió a cuantificar el índice de representación anatómica (en adelante, IRA), siguiendo la propuesta de Bello y Andrews (2006) que relaciona el número de huesos recuperados (NHR) y el de aquellos que cabría esperar (NHE) en atención al número de individuos. El valor resultante aporta información diversa que abarca desde la cualificación de la representación anatómica (buena cuando el IRA supera el 50\%) hasta la estimación de qué fenómenos naturales o antrópicos intervienen en cada contexto, con qué intensidad y sentido.

En el caso de la mujer el IRA contabilizado puede considerarse bueno, pues alcanza casi el 59\%, mientras que en el subadulto se reduce significativamente al situarse en un 34,9\% (Fig. 3). Por ejemplo, en este segundo caso solo el $15 \%$ de los metacarpos/metatarsos y $5 \%$ de falanges están representados, faltando todos los carpos. Asimismo, están ausentes, entre otros elementos, el esternón, varias vértebras dorsales y cervicales, el pie izquierdo y el brazo derecho. Es cierto que la ausencia de huesos de pequeñas dimensiones (falanges, carpos, etc.) provoca un índice que puede resultar en apariencia pequeño, pero de cualquier modo es un buen testimonio de la representación parcial de este individuo y, en particular, de un IRA más bajo que el calculado para la mujer. Esta, por su parte, ha conservado el 14,3\% de los tarsos, el 25\% de los metacarpos/metatarsos o el $42 \%$ de las falanges, destacando la ausencia de tibia, peroné, ambos pies, así como algunas vértebras. La explicación a la subrepresentación anatómica y la disparidad en el IRA exige analizar otra evidencia presente en los dos individuos: las marcas originadas por la acción de carnívoros en el registro óseo. 


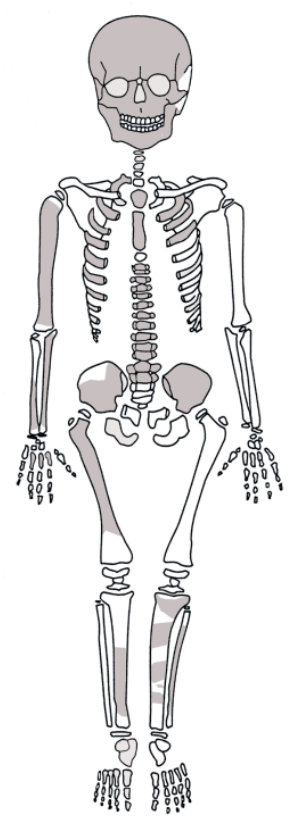

Fig. 3. Esquema de la representación anatómica del subadulto.

\subsubsection{Huesos mordidos}

La evidencia de carroñeros sobre ambos esqueletos es muy clara, como así se constata, entre otros marcadores, por la presencia de fracturas, impactos dentales, surcos en las diáfisis y, en especial, dentelladas con hundimiento de la cortical y los característicos bordes festoneados en las epífisis y huesos planos. Si se observan en detalle la morfología, localización y dimensiones de tales alteraciones es posible atribuirlas, en uno y otro individuo, a cánidos (Colard et alii, 2014; Madgwick, 2008), y la configuración, distribución e intensidad de tales trazas ayudan a entender el modo en que se produjeron y su responsabilidad en el estado final del depósito.

En el subadulto, las huellas provocadas por carnívoros están mucho más generalizadas que en la mujer, lo que explica el menor IRA al que se aludía, destacando en este sentido la intensa alteración en el brazo derecho. Así, el húmero, al que faltaba una porción central de la diáfisis, muestra ambos extremos con el característico patrón de dentelladas en el hueso trabecular y bordes aserrados en el compacto (Fig. 4). El cúbito de este lado exhibe una afección similar, con la destrucción parcial de ambas epífisis (Fig. 5). Tanto en el cúbito como en el radio se aprecia que los extremos colapsan por la presión 
ejercida por las mordeduras de estos carnívoros, produciendo los ya reseñados bordes festoneados, con la presencia de fragmentos de tejido compacto hundidos y pequeñas fracturas de desarrollo longitudinal.

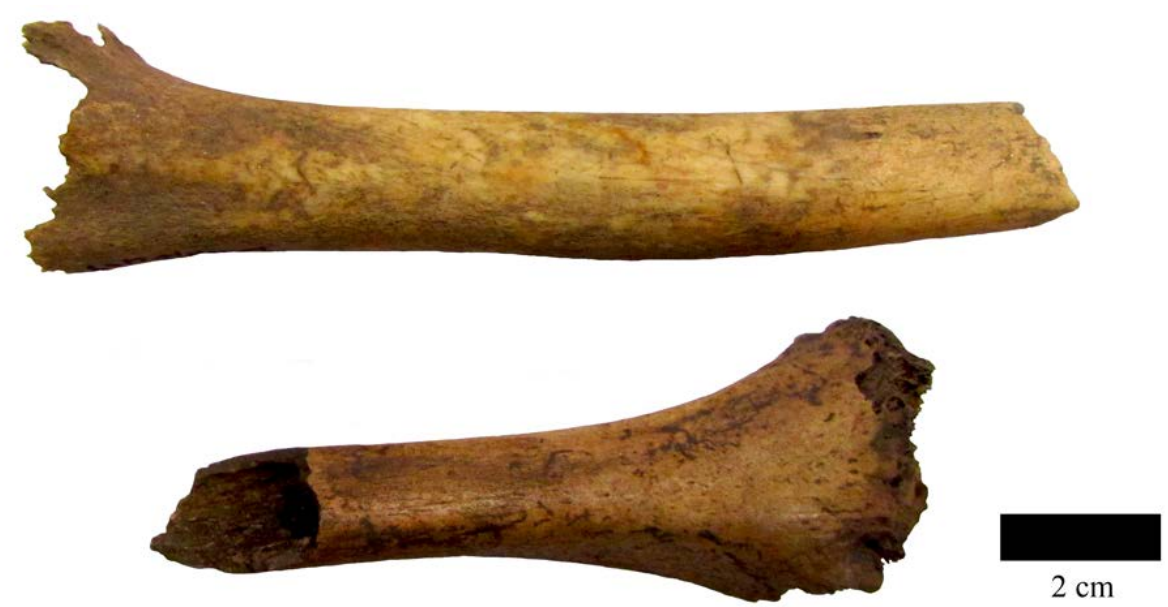

Fig. 4. Húmero derecho con evidencias de la acción de carnívoros en ambos extremos.

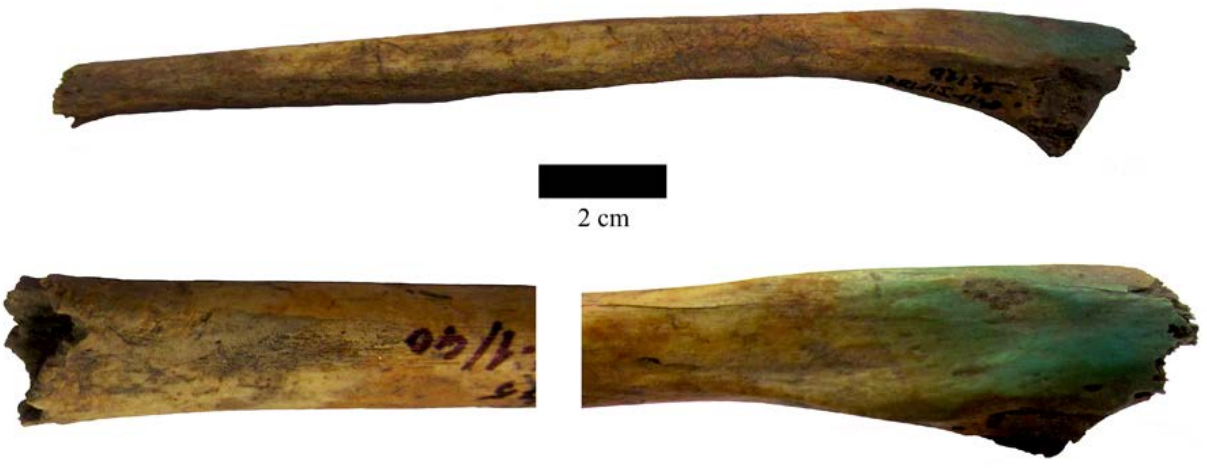

Fig. 5. Cúbito derecho con ambas epífisis afectadas por mordeduras.

En cuanto a la cintura pélvica, las trazas dejadas por los animales son también muy características, sobre todo en el sacro y las porciones iliacas. El mayor daño se registra en la región posterior de la espina iliaca, así como en las superficies auriculares (Fig. 6). Además de la destrucción de hueso, en sendas 
palas son muy evidentes las dentelladas que, en algún caso, se registran pareadas dejando una huella circular en el hueso esponjoso y el hundimiento del compacto, aún visible en el interior de la mordedura (Fig. 7). En el fémur derecho, único presente, se observa una intensa destrucción de la zona que comprende el trocánter mayor y, en relación con ello, la presencia de surcos de escaso recorrido y poco profundos en la zona anterior del tercio proximal de la diáfisis (Fig. 8). Las tibias son, sin duda, las porciones esqueléticas más afectadas por este tipo de alteraciones, pues a las consabidas marcas se suma la fragmentación siguiendo el patrón de fractura habitual en los huesos largos afectados por la acción de carroñeros (Binford, 1981; Sala et alii, 2014; Parkinson et alii, 2014). En diferentes puntos de la diáfisis de ambas tibias —en especial en las áreas más subcutáneas- se aprecian surcos debidos a la acción repetitiva de los dientes sobre las cañas (Fig. 9).

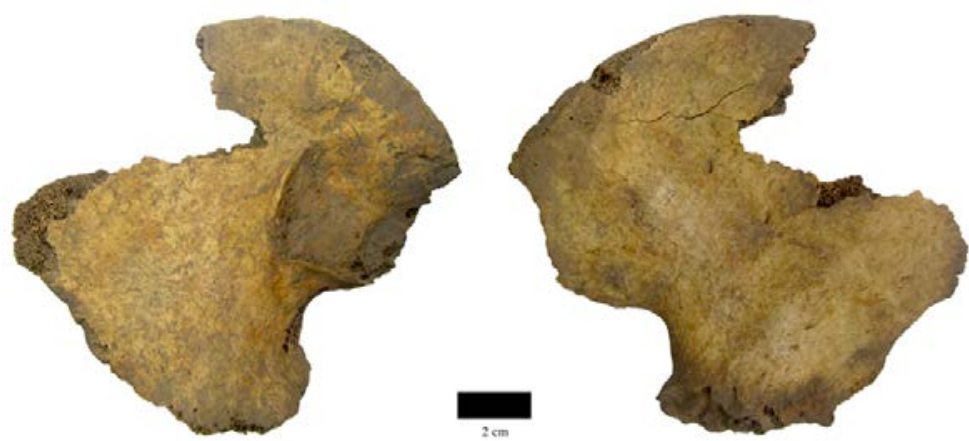

Fig. 6. Coxal derecho.
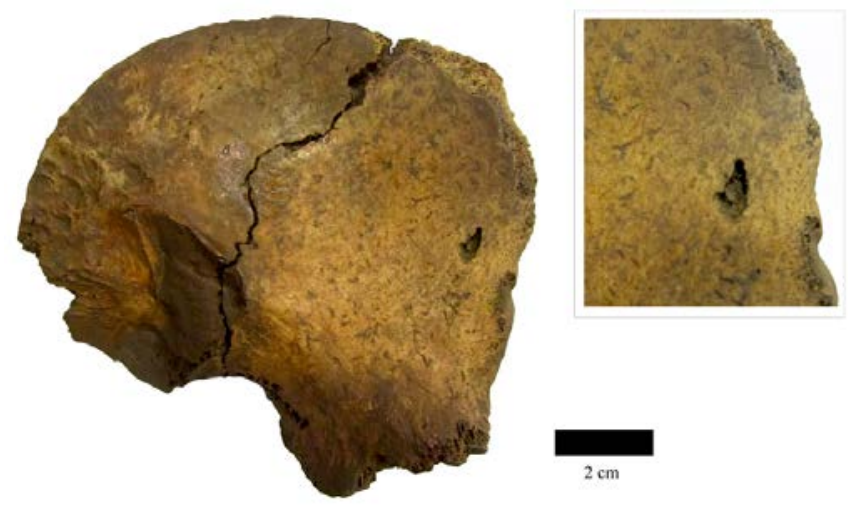

Fig. 7. Coxal izquierdo con impacto pareado. Recuadro superior derecho: detalle de impacto de canino. 

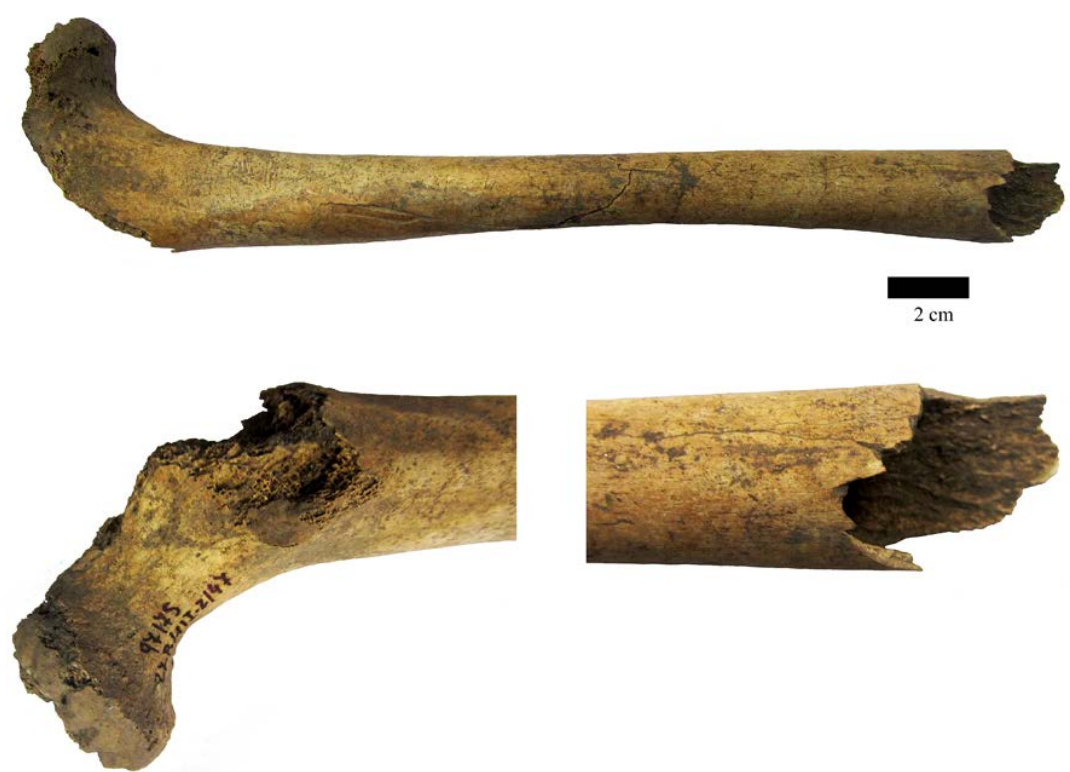

Fig. 8. Fémur derecho. Arriba, vista anterior. Abajo izquierda, vista posterior de tercio proximal. Abajo derecha, detalle del extremo distal.
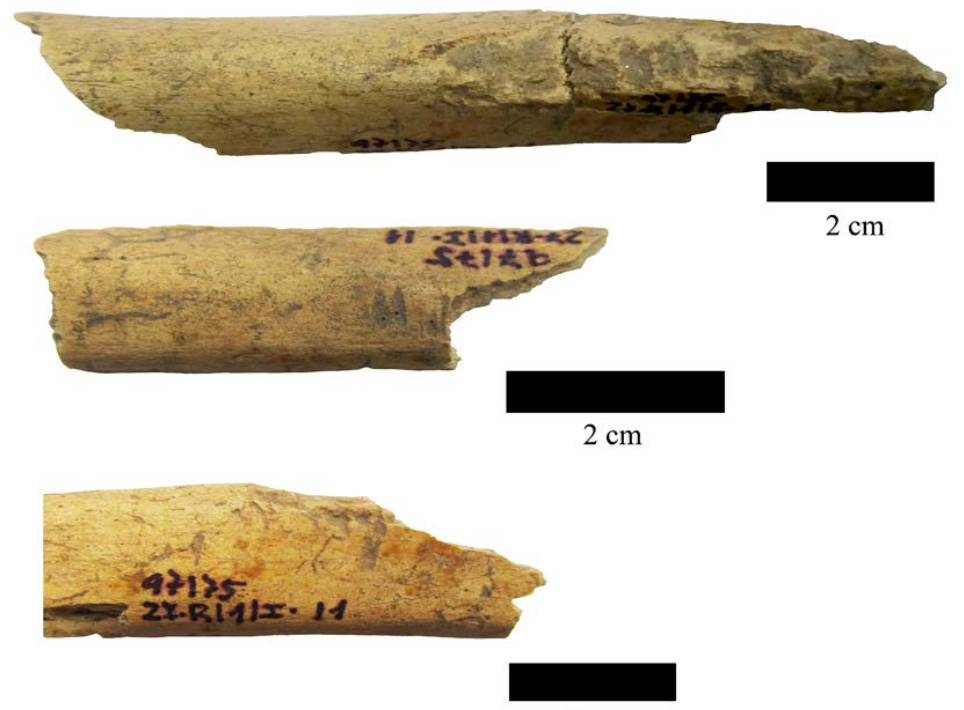

$2 \mathrm{~cm}$

Fig. 9. Fragmentos de diáfisis de ambas tibias con impactos y surcos atribuibles a la acción de cánidos. 
Finalmente, hay que destacar que la acción de cánidos, además de en el esternón, también se registra en el lateral izquierdo del cráneo (occipital, parietal y temporal), el atlas y, de forma muy evidente, en la rama ascendente izquierda de la mandíbula (Fig. 10). En esta última las dentelladas provocaron la pérdida de importantes porciones de hueso, lo que no sucede en el lado contrario.

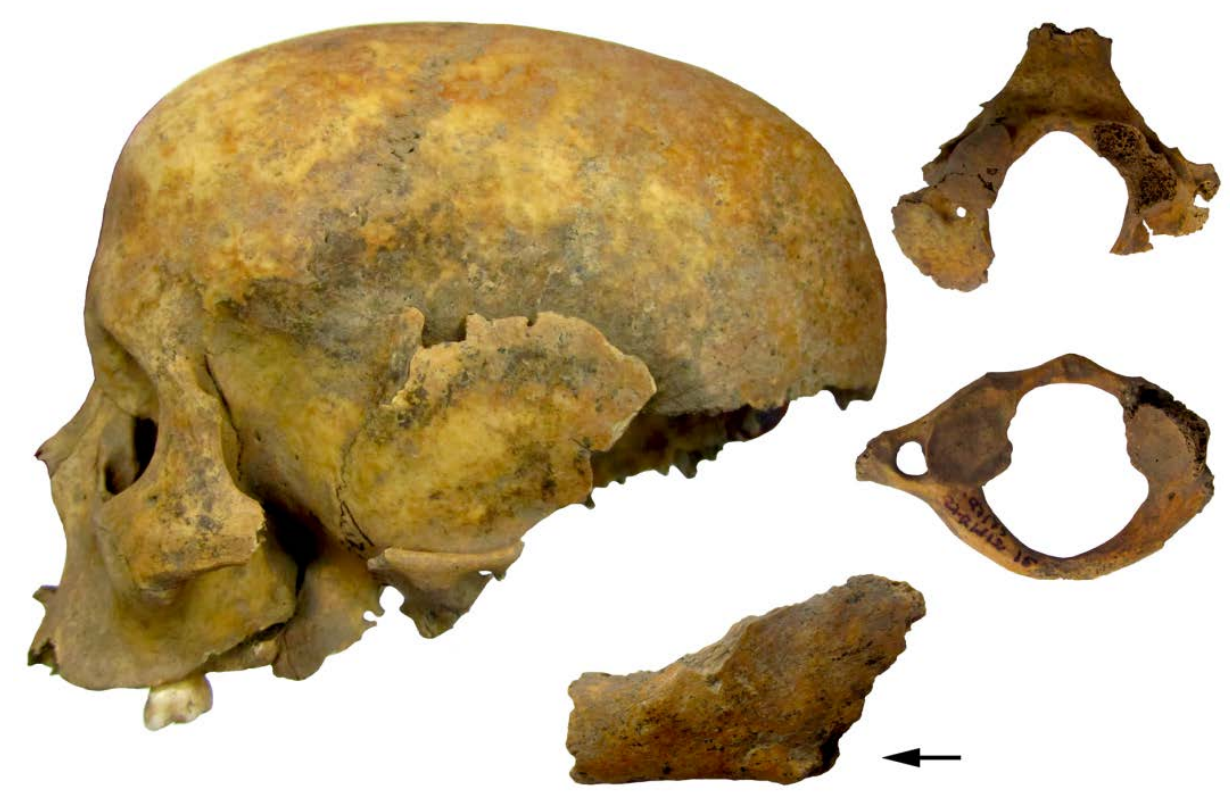

Fig.10. Vista lateral izquierda del cráneo, porción de la base del cráneo, atlas y fragmento del lateral izquierdo de la mandíbula (flecha)

El mismo fenómeno de intervención de carnívoros se reitera en el esqueleto de la mujer. Así, en su húmero izquierdo presenta ligeras modificaciones en la extremidad distal (epicóndilo medial), mientras que la epífisis proximal ha sido parcialmente destruida. El derecho solo conserva parte del tercio distal, faltándole una porción de la tróclea y el epicóndilo (Fig. 11). También fueron alterados el cúbito (Fig. 12) y radio derechos, con la completa desaparición de la epífisis proximal del segundo y una destacada alteración del primero. Además, la escápula del mismo lado muestra signos de mordeduras localizadas en el borde inferior y en el ángulo superior, donde se aprecian los impactos de las piezas dentales y el distintivo borde aserrado (Fig. 13). 


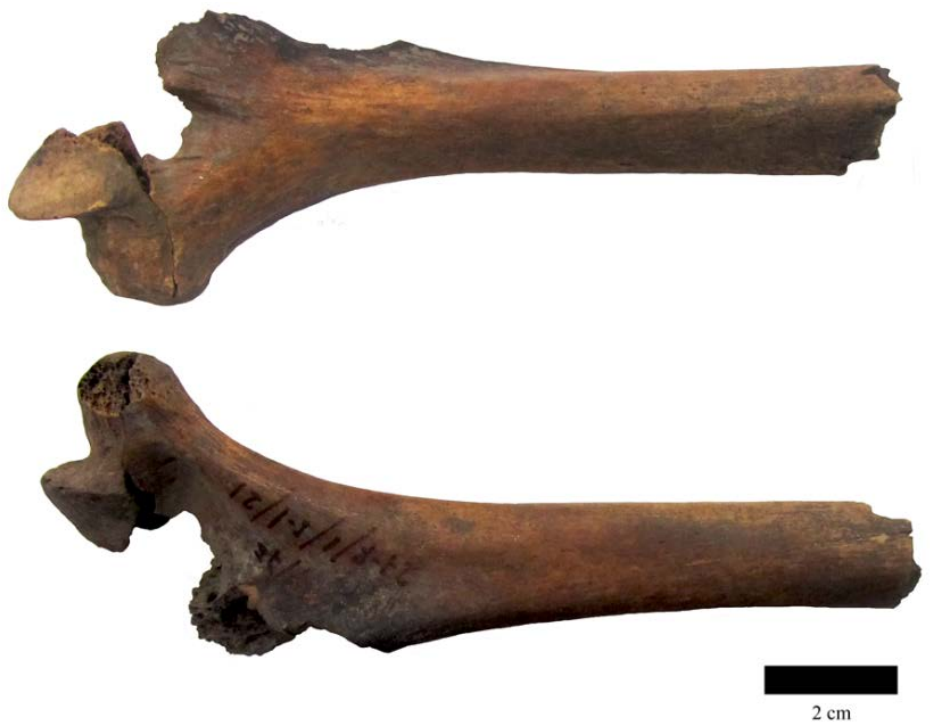

Fig. 11. Mitad distal de húmero derecho (vista posterior y anterior).
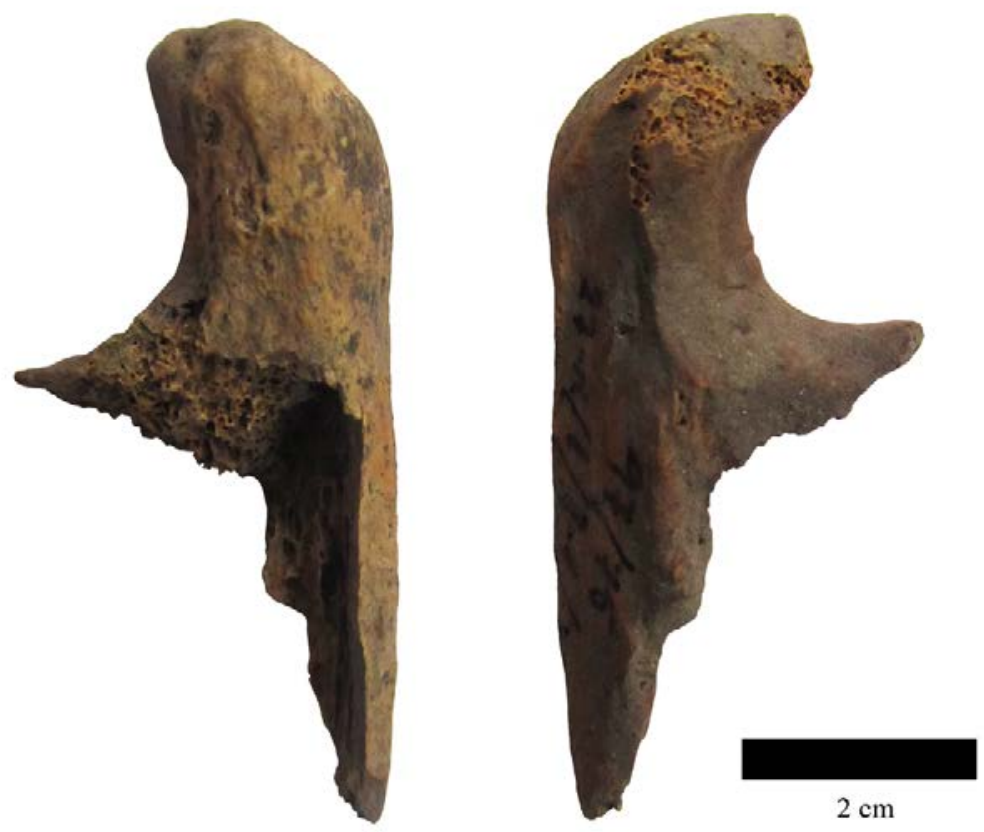

Fig. 12. Epífisis proximal cúbito derecho (vista medial y lateral) 

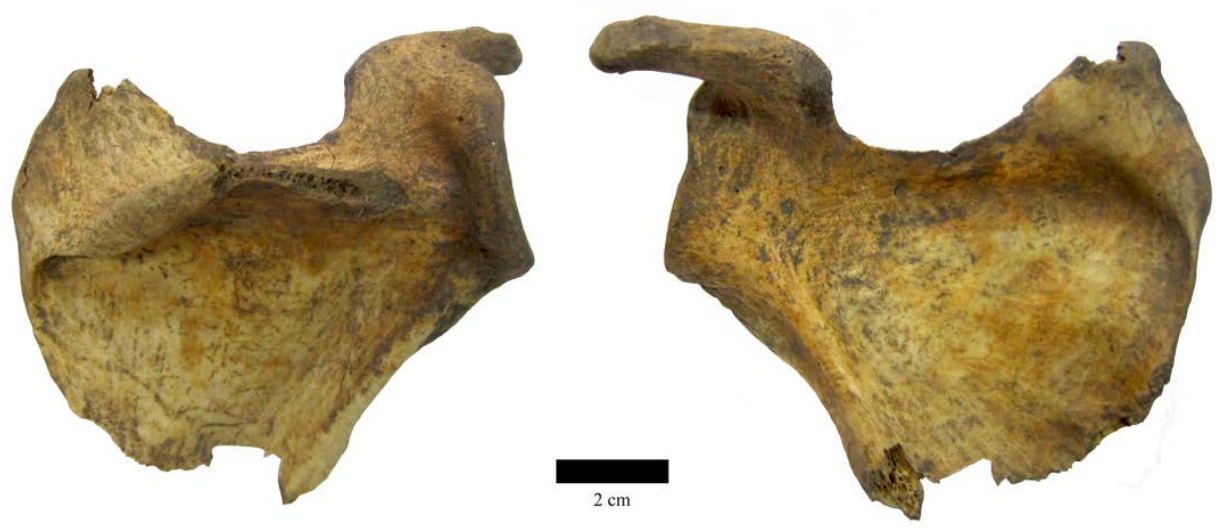

Fig. 13. Escápula derecha (vista posterior y anterior)

En este individuo, las mayores modificaciones de origen animal se localizan en la región lumbar, cintura pélvica y zona proximal del fémur derecho. Las apófisis transversas y espinosas de L2 y L3, así como las superficies auriculares y las crestas laterales del sacro, especialmente a partir de S2, exhiben huellas inequívocas de mordeduras que conllevaron la pérdida de una porción significativa de hueso. En ambos coxales también es muy perceptible esta situación, en especial en la cresta iliaca, la espina iliaca superoposterior y la tuberosidad isquiática (Fig. 14). Ahí se identifican huellas de dentelladas en el hueso trabecular, que en la cortical se traducen en bordes aserrados preferentemente localizados en la zona posterior de los coxales, más acentuado en el derecho que en el izquierdo. El fémur diestro muestra una intensa acometida en la región proximal de la que solo queda indemne la cabeza, protegida al estar inserta en el acetábulo, mientras que la región correspondiente a ambos trocánteres se destruyó casi por completo, afectando también a parte de la diáfisis (Fig. 15). En el fémur izquierdo la presencia de este tipo de marcas se limita a afecciones puntuales.

Las huellas de animales en los dos esqueletos permiten plantear que los carroñeros — perros o lobos a juzgar por el patrón observado- tuvieron un acceso primario a los cuerpos. En otras palabras, su actuación se produjo cuando los huesos todavía se encontraban cubiertos por tejido blando, probablemente porque el cadáver estaba fresco o en un estadio inicial de la descomposición. En estas situaciones, y como sucede en el Cerro de la Cabeza, las huellas en los huesos no son muy abundantes, observándose algún surco en las diáfisis, pero sobre todo dentelladas y bordes aserrados en los extremos de los huesos largos y los planos (Binford, 1981; Smith, 2006). Trazas que se 
distinguen de aquellas en que las carcasas óseas están al alcance de los animales durante un prolongado período de tiempo, lo que permite una interacción muy intensa (Binford, 1981; Haglund, 1997; Haynes, 1980, 1983; Smith, 2006). En esta misma línea, es muy probable que pueda achacarse a la acción de los carroñeros la desaparición de parte de los restos esqueléticos, bien porque fueron directamente ingeridos, bien al ser separados del cuerpo y trasladados a otros lugares por estos animales (Fosse et alii, 2012; Camarós et alii, 2013). El reducido número de huesos de pies y manos (fundamentalmente falanges, metacarpos y metatarsos, carpos o tarsos) es compatible con la propuesta de un carroñeo primario, al ser las zonas que, por lo general, resultan más accesibles y fáciles de desprender por parte de los animales (Binford, 1981; Colard et alii, 2014). Como también lo es que en las pelvis sean las zonas iliacas y la tuberosidad isquiática las regiones más frecuentemente dañadas, además de los márgenes del sacro, extremos de los húmeros, cúbitos y radios, así como las escápulas (Haglund, 1997: 374-375).

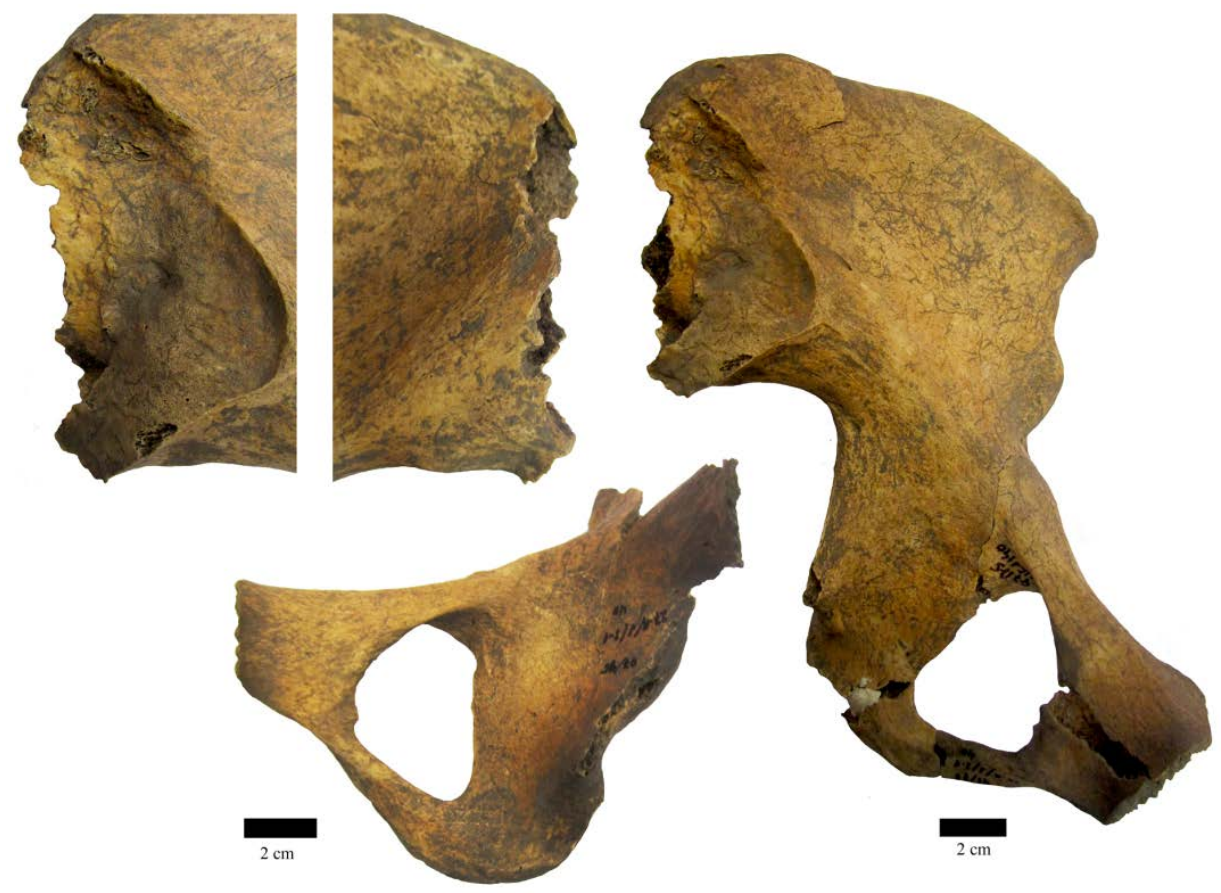

Fig. 14. Coxales. Arriba, a la izquierda: vista medial y lateral de la región auricular. 


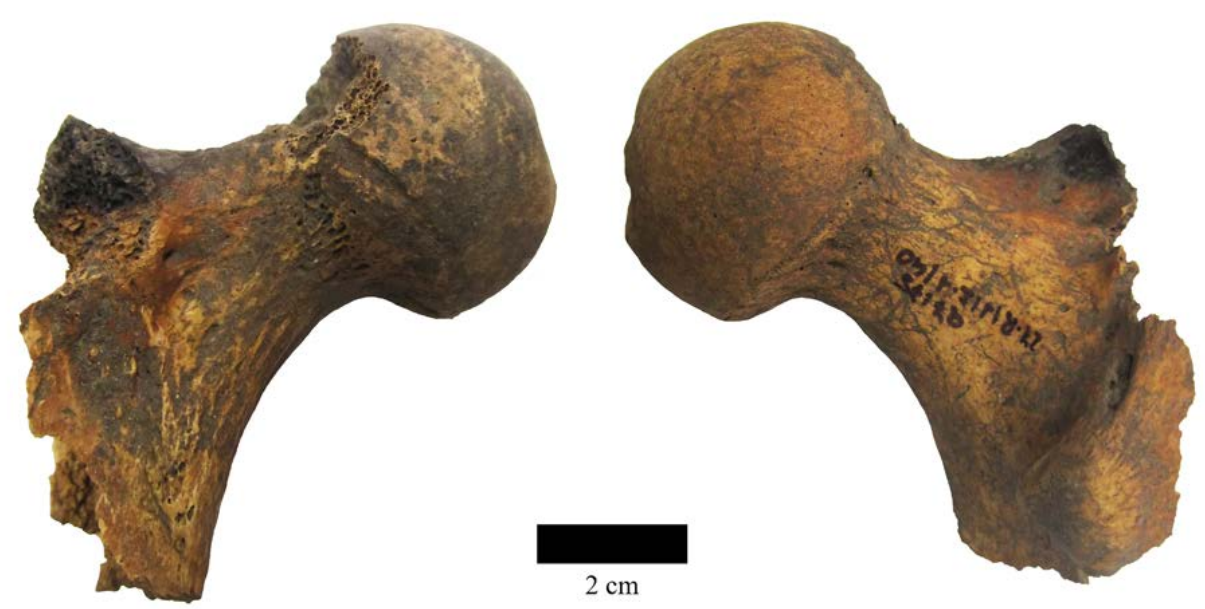

Fig. 15. Epífisis proximal fémur derecho (vista anterior y posterior)

Como se recoge en diversos trabajos (Faith et alii, 2007; Haglund, 1997; Madgwick, 2008), las variables que condicionan la zona del esqueleto y la intensidad con la que se ven afectadas las piezas óseas -la propia configuración anatómica del hueso, la arquitectura/configuración del lugar de acogida, la presencia de sedimentos o piedras en su interior, el número de animales que participan, la posición del cuerpo, etc. - contribuyen a reconstruir los procesos observados. En nuestro caso, parece clave la forma en que los cuerpos fueron acomodados dentro del hoyo, habiendo indicios reveladores en ambos esqueletos. En la mujer, un porcentaje elevado de las afecciones imputables a los animales se localiza en la parte posterior de las piezas óseas, precisamente la que quedaría más expuesta con el cadáver en decúbito prono. Tal circunstancia se aprecia con nitidez en la cintura pélvica y en el fémur derecho, donde una vez eliminada la cobertura de tejido blando que cubre estos huesos quedaron accesibles las porciones en las que se identificaron las afecciones: región posterior de la cresta iliaca, espina iliaca, crestas laterales del sacro, trocánter menor, etc. En el sujeto infantil, el patrón de acceso puede analizarse en términos semejantes, de modo que las huellas se sitúan en la zona anterior del cuerpo, coincidiendo con la disposición de decúbito supino, tal y como se deriva de las dentelladas y surcos en las crestas tibiales, en el esternón, la zona anterior de las pelvis, en algunas porciones esternales de las costillas, etc.

La intensidad y localización de las huellas en cada cuerpo revela exposiciones diferentes en uno y otro caso. Tanto la menor representación anatómica, como las huellas en el registro esquelético ayudan a sostener que el 
subadulto debió de experimentar durante más tiempo la acción de los animales, en particular en su extremidad inferior. En este sentido es de interés acudir a la información que proporcionan las tablas de tiempo de descarnado y desarticulado de cadáveres por cánidos elaboradas por Haglund (1997), pues según este autor el proceso tiene lugar en una secuencia relativamente constante que permite la propuesta de unos intervalos que se han revelado de utilidad en los trabajos de antropología forense (Steadman y Worne, 2007; Kjorlien et al., 2009; Moraitis y Spiliopoulou, 2010), y en los bioantropológicos (Smith, 2006; Madgwick y Mulville, 2015). Atendiendo a lo expuesto en dichas tablas, el acceso de los cánidos a los cuerpos del Cerro de la Cabeza puede situarse entre el estadio 1 (evisceración, alteración de los huesos del tórax, clavículas, separación de una o las dos extremidades superiores, y su traslado a veces) y el 2 (cuando comienza la disociación de las extremidades inferiores, muchas veces a partir de los cóndilos femorales). Al final del estadio 1 - la franja en la que probablemente se sitúa al menos la mujer adulta - la mayor parte de los músculos de las zonas pélvicas, torácicas y los muslos han sido consumidos, quedando expuestos los huesos de estas regiones (Haglund, 1997: 369). Traducido en tiempo, el estadio 1 se fija en un intervalo que va entre 22 días y 2,5 meses, mientras que el 2 se situaría entre 2 y 4,5 meses. Son unos márgenes temporales que han de entenderse orientativos, pero que permiten concretar el fenómeno del acceso a los cuerpos por parte de los carroñeros durante un tiempo concreto.

Teniendo en cuenta lo expuesto hasta el momento, la valoración de los daños registrados en uno y otro individuo contribuye a clarificar el orden de los procesos que configuran el depósito. Como ya se ha dicho, el brazo derecho del subadulto, único que conserva, presentaba evidentes signos de dentelladas en los extremos del húmero, cúbito y radio. Precisamente esta región anatómica fue cubierta por el cadáver de la mujer cuando fue introducido en el hoyo, por lo que a partir de ese instante estaría a salvo de la acción de los cánidos. En otras palabras, en un primer momento se depositó el cuerpo del subadulto en el fondo del hoyo, quedando expuesto a la acción de los carroñeros. Tiempo después se introdujo la mujer, resultando a partir de entonces accesible a los animales, condición que también mantuvo el subadulto, salvo en las zonas cubiertas por el nuevo cadáver. Esta diferencia en los tiempos de deposición ayuda a entender que el material esquelético del sujeto de menor edad muestre daños de mayor alcance que el de la mujer y que, a la par, cuente con un índice de representación anatómica más bajo, pues estuvo sometido a la acción carroñera durante un período más largo.

Es muy difícil concluir cuánto tiempo pudo pasar entre la introducción de uno y otro cadáver. A juzgar por el daño observado en el subadulto cabe suponer que rondaría entre unas pocas semanas y dos meses, como se desprende de las modificaciones en el brazo derecho imputables a los carroñeros adscritas 
a la fase 1 de Haglund (1997), mientras que el izquierdo ha desaparecido por una mayor exposición. Pero más allá de lo certero de tal estimación temporal, se deduce que la exposición de los cadáveres en el hoyo —o al menos el hecho de que fueran depositados de forma tal que resultaran accesibles a los animales durante un determinado lapso temporal - difícilmente puede tildarse de accidental. El primer cuerpo se sitúa en el hoyo y no se tapa, o se hace con algún elemento lábil, pero cuando tiempo después se introduce el segundo, se mantienen las condiciones de accesibilidad a pesar de que las consecuencias de la acción de los animales sobre el subadulto eran evidentes. Esto es, se reitera una acción a sabiendas de lo que allí sucede. No parece que pueda hablarse de un espacio funerario en el cual, por descuido o por un cierre poco esmerado, se colaron los animales. Resulta más verosímil el que los cuerpos, primero uno y luego otro, quedasen conscientemente expuestos en un espacio abierto o semiabierto, sin que se pueda asegurar que el fin perseguido era que los cánidos participasen activamente en el descarnado.

Finalmente, debe destacarse la más que posible responsabilidad de los carroñeros en el desplazamiento de ciertos huesos: la coincidencia de piezas que se encuentran alejadas del lugar que debieron de ocupar en el depósito inicial y que, a la vez, muestran signos evidentes de dentelladas y mordeduras es un buen indicio en este sentido, como así podría ejemplificar la mandíbula del subadulto, localizada junto al cráneo del adulto.

\subsubsection{Huesos quemados}

Como ya se había señalado en el primer trabajo sobre del Cerro de la Cabeza (Fabián et alii, 2010: 186-188), los restos presentan signos de alteración térmica, lo que también contribuye a explicar la secuencia de actos que dan lugar al depósito. Al igual que se ha descrito para la mordeduras, las afecciones atribuibles al calor muestran diferencias entre los dos sujetos. En la mujer estas alteraciones post mortem son muy evidentes en el flanco derecho del cuerpo, en particular en el brazo y costillas de este lado, así como en el coxal y el proximal del fémur (Fig. 16). En las zonas en las que el calor fue más intenso — proximal de fémur, cresta iliaca derecha, algunas porciones esternales de costillas, distal de húmero y proximal de cúbito-, el tejido óseo muestra el característico color negro propio de los estados de carbonización. En el resto del esqueleto, la coloración va desde marrón a ocre, con variaciones graduales perceptibles incluso dentro del mismo hueso, si bien siempre con más intensidad en la zona posterior y lateral de los huesos dañados. En cuanto al subadulto, la modificación térmica se identifica preferentemente en su mitad superior y, en especial, en el flanco derecho, como así sucede en parte del cráneo, húmero, cúbito y radio, algunas costillas, lateral de la mitad proximal del fémur derecho 
y el ilion de este lado. Las variaciones de color son menos acusadas que en el caso anterior y, por lo general, puede hablarse de una gama de anaranjadosocres. En este sentido, destaca el que parte de los huesos de ambos individuos muestren una apariencia brillante y una coloración que recuerda a la que se describe para los huesos "asados" (Botella et alii, 1999; Trujillo et alii, 2015), sintomático de la presencia de materia orgánica en el momento de la termoalteración.
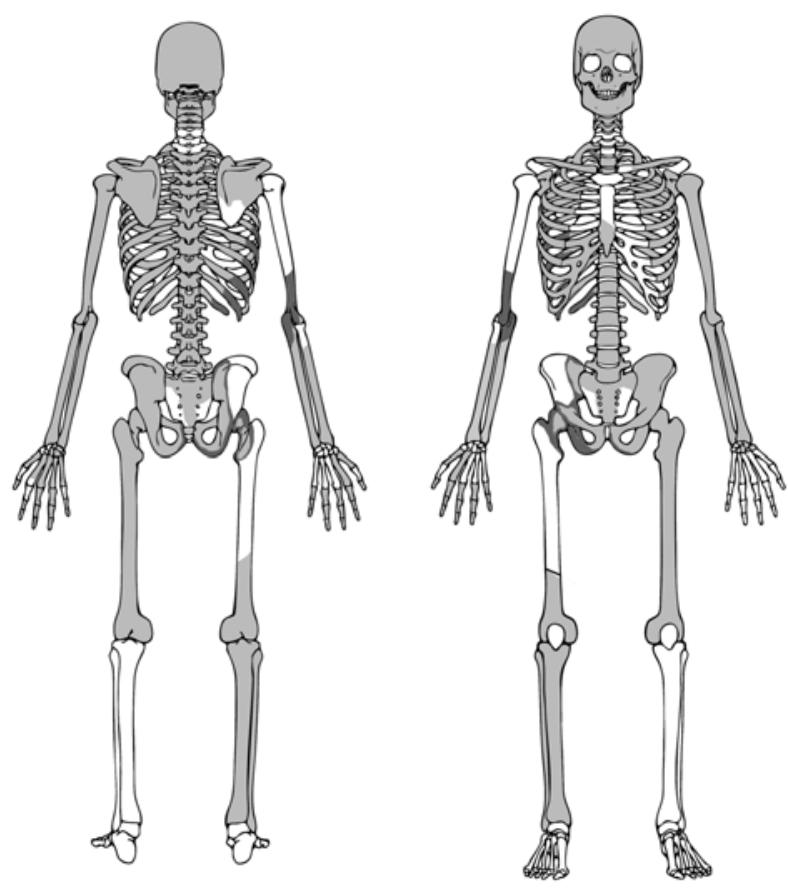

Fig. 16. Representación anatómica en el individuo femenino. En gris más oscuro, áreas afectadas con mayor grado de termoalteración

Como se desprende de la dispar localización de las alteraciones térmicas en uno y otro individuo, el fuego les habría afectado cuando ambos estaban en el hoyo y en una posición semejante a la registrada en en el curso de la excavación arqueológica. El foco principal de calor debió de localizarse aproximadamente a la altura de la cadera derecha de la mujer, pues es en esta zona — pelvis, costillas, distal de húmero, proximal de fémur - donde los huesos revelan haber alcanzado una temperatura más alta, entre $300-350^{\circ} \mathrm{C}$ (Etxeberria, 1994; McKinley, 2000; Walker et alii, 2008). El calor irradiado desde este punto afectó con una intensidad gradual a los huesos de ambos individuos (según 
distancia y disposición), alcanzándose una temperatura que pudo oscilar entre $200-250^{\circ} \mathrm{C}$, muy probablemente sin que se vieran afectados directamente por las llamas. El fuego, no solo está atestiguado por la alteración térmica que muestran los huesos, sino que asimismo parece confirmarse en la localización que se propone por la coloración de los sedimentos, según se aprecia en la documentación gráfica de la excavación y que concuerda con la distribución de las huellas en los restos de ambos individuos. De ahí que en la mujer, la termoalteración, aunque generalizada, afecte más al lado derecho que al izquierdo y, de igual modo, al flanco derecho del subadulto, por su mayor proximidad al fuego. Asimismo, los testimonios de combustión son más intensos en la mujer que en el niño producto también de la distancia al foco.

En apariencia la acción del fuego fue posterior a la afección imputable a los cánidos, pues las alteraciones térmicas se identifican en aquellas porciones de hueso con huellas de mordeduras preexistentes. Esta circunstancia se aprecia muy bien en las semejanzas de coloración en el tejido compacto y trabecular, así como en los bordes dejados por las dentelladas en diferentes huesos, siendo buen ejemplo de lo dicho lo registrado en el distal del húmero y en el proximal del fémur. En este mismo sentido, las alteraciones registradas en pelvis y fémur, bien protegidas por una densa masa muscular en un cadáver fresco, y las del distal del húmero, más subcutánea, no podrían haber sido tan semejantes entre sí a no ser que las primeras ya hubieran quedado previamente expuestas por los carroñeros. Con todo, no puede descartarse que, tras el fuego, los animales siguieran frecuentando los cadáveres y provocaran algún daño a los huesos, pero es una eventualidad más complicada de estimar. Finalmente hay que apuntar que el fuego también afectó a los restos de fauna identificados en el fondo del hoyo, con algo más de intensidad en lo que respecta a la mandíbula que al húmero (Fig. 17).

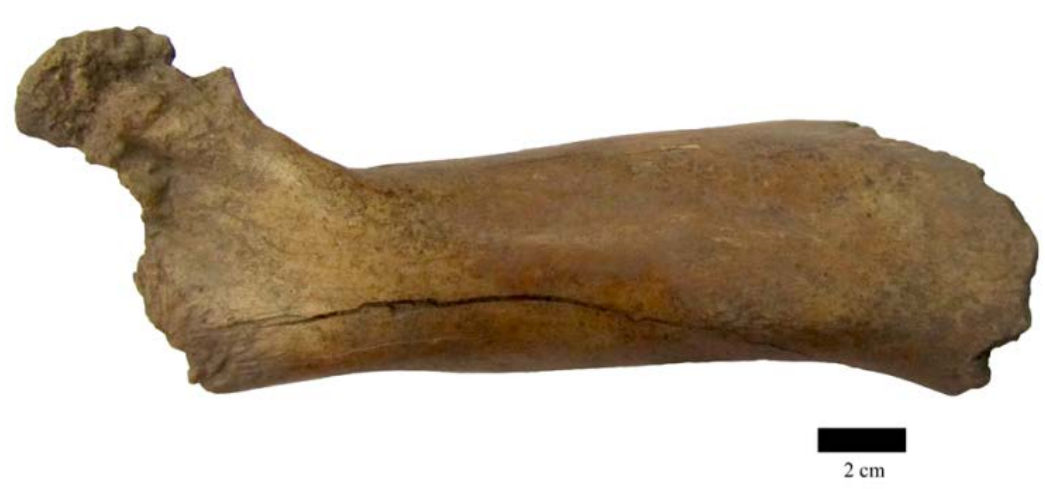

Fig. 17. Fauna afectada por los mismos procesos tafonómicos que los descritos para los humanos. 
Recapitulando lo expuesto hasta el momento, la formación del depósito del Cerro de la Cabeza podría concretarse de la siguiente manera: en primer lugar, se introduce el cadáver de un subadulto de unos 11-13 años que, al no cerrarse el hoyo, queda al alcance de cánidos que comienzan a alimentarse de su cuerpo y probablemente a desmembrar ciertas regiones anatómicas (Fig. 18A). Con posterioridad - entre unas semanas y un par de meses - se incorpora el cuerpo de una mujer adulta, que a partir de entonces también es accesible a la acción de los carroñeros, responsables de las huellas de mordeduras reconocibles en su esqueleto y muy posiblemente de la ausencia de ciertos huesos (Fig. 18B). Seguidamente se hace en el interior del hoyo un fuego que, aún no alcanzando gran poder calorífico, sí afectó a los huesos de ambos individuos cuando ya se encontraba avanzado el proceso de esqueletización (Fig. 18C). Por último, el hoyo es cubierto de tierra y piedras, impidiendo el movimiento de los huesos que aún mantenían conexiones anatómicas, y sobre todo, que los cuerpos siguieran estando francos para los animales (Fig. 18D).
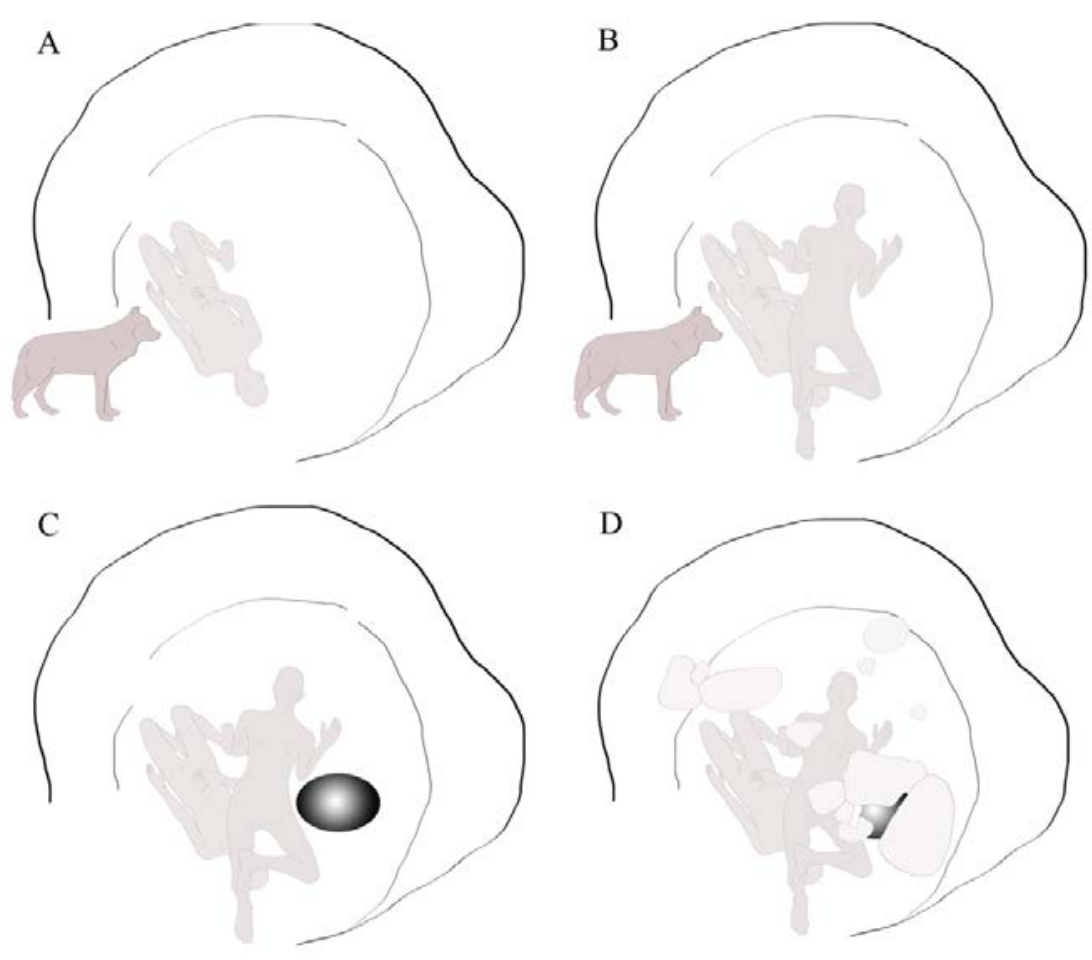

Fig. 18. Resumen gráfico de la propuesta de secuencia de formación del depósito. 


\section{CONSIDERACIONES FINALES}

Aunque en la imagen arqueológica no resulte del todo perceptible lo que cabría esperar de una colocación preconcebida, a la vista del contexto puede defenderse la existencia de una disposición cuidada de los cuerpos. $\mathrm{O}$ al menos que, como en otros casos, se realizó una composición mortuoria que atañe a ambos cadáveres, y quizá a la introducción de otros elementos como la fauna. Un cuerpo es situado directamente sobre el otro, con orientaciones y posiciones contrarias, sin que exista un condicionante físico que así lo obligue, ajustándose, al menos en apariencia, a un esquema que define cómo ha de ser colocado cada individuo y cuál debe ser el aspecto resultante de tal gesto. El primer cadáver queda accesible a los carroñeros al ser depositado en el hoyo, lo que trae aparejadas unas consecuencias que son evidentes cuando allí se introduce la mujer adulta, lo que sin embargo no se traduce en medidas que eviten tal situación. El depósito se conforma en diferentes momentos, como resultado de distintas acciones ligadas entre sí, no solo por el corto lapso de tiempo -en sentido deposicional- que media entre ellas, sino por la propia naturaleza de la práctica mortuoria en la que es raro que algunos de los gestos queden al azar. Parece, pues, que se busca dejar insepultos los cuerpos hasta un momento avanzado de su esqueletización, si bien es difícil saber si con ello se pretende facilitar un descarnado activo de los cadáveres con la ayuda de los animales o si la eventual intervención de éstos era vista como un daño menor, que no afectaba a lo que representaba la escenificación de la muerte de esas dos personas. En este sentido, cabría reflexionar sobre el papel del fuego, para el que de momento no es posible conocer si se trata de un componente ritual o más bien un mero aspecto práctico, quizá destinado a ahuyentar a los animales que frecuentan el hoyo.

La presencia de huellas de carnívoros en restos humanos de Cogotas I no es exclusiva de este yacimiento. Quizás el ejemplo más significativo sea el salmantino de Tordillos en Aldeaseca de la Frontera (Esparza et al., 2012b), en el que los restos óseos de dos individuos mostraban huellas de mordeduras, además de claros signos de exposición a la intemperie. El análisis de estos materiales, correspondientes a sendos depósitos secundarios, ayudó a plantear la hipótesis de que mientras los entierros en hoyos podían responder a eventos excepcionales, la exposición de cadáveres habría sido la norma funeraria de este periodo. En algunos casos, y con criterios desconocidos - tal vez con ocasión del abandono de un asentamiento-, parte de los cuerpos así expuestos eran seleccionados y enterrados luego en hoyos, bien solos, bien acompañando otros depósitos primarios. De este modo, las diferencias entre Tordillos y lo registrado para el Cerro de la Cabeza son evidentes. En la muestra abulense el repertorio óseo no tiene huellas atribuibles a los agentes ambientales pero sí de 
fuego, ausente en el yacimiento salmantino. En segundo lugar, el de Ávila manifiesta un carácter primario y la acción de los animales parece bastante más limitada, mientras que los depósitos de Tordillos fueron realizados en dos o más tiempos, pasando los cuerpos una temporada a la intemperie para después ser introducidos en el hoyo, siendo en ese primer episodio cuando los animales pudieron haber entrado en contacto con los cuerpos. En tercer lugar, también parecen diferentes los tiempos fúnebres, siendo más largo el periodo de exposición en el caso de Tordillos, como parece desprenderse de la intensidad de las marcas. En definitiva, se evidencian dos modelos que tienen en común la participación de los animales, pero que, a partir de la información actualmente disponible, difieren bastante en otros componentes de la práctica mortuoria.

Además de la presencia, o la constatación, de huellas de carnívoros en los restos óseos humanos, otros indicios pudieran considerarse testimonios indirectos de la participación de estos animales en la conformación de los depósitos de Cogotas I. Así, la dispersión aparentemente caótica de huesos en el interior de los hoyos o la subrepresentación anatómica, en particular de ciertos elementos de menor entidad, bien pudieran ser el resultado de la acción de los carroñeros. Lo mismo puede decirse de determinados depósitos, valorados como primarios, en los que recurrentemente coincide la ausencia de elementos anatómicos y el desplazamiento de, por ejemplo en Los Berrocales (Aliaga y Megías, 2011) o en el Sector 22 de Yuncos (Barroso et alii, 2014), que, a priori, concuerdan con lo descrito para el Cerro de la Cabeza.

Llegados a este punto, la evidencia disponible nos sitúa ante esta disyuntiva:

a) El caso del Cerro de la Cabeza sería asimilable a otros lugares donde debía de realizarse la exposición que ha sido propuesta como práctica general en Cogotas I.

b) Alternativamente, el depósito del Cerro de la Cabeza sería, con sus particulares características, una más de las sepulturas en hoyo relacionadas con la 'mala muerte'.

En todo caso, cabría preguntarse también: ¿se trata de un espacio donde los cadáveres fueron abandonados a su suerte durante cierto tiempo para luego ser sepultados? ¿Se buscaba intencionalmente el acceso de los animales a los cadáveres? ¿Hay en estas conductas un gesto de reprobación o rechazo social?

La disyuntiva planteada queda forzosamente sin resolver, en buena medida porque la respuesta a tantas cuestiones pasa, casi ineludiblemente, por establecer si el Cerro de la Cabeza es un caso único o si, por el contrario, hay otros contextos semejantes. Pero de lo que no cabe duda es que los sucesivos hallazgos corroboran la complejidad y diversidad de las expresiones arqueológicas en los contextos de Cogotas I con restos humanos. A la vista de todos ellos, sigue siendo muy difícil ofrecer una única solución a la amplia 
casuística mortuoria registrada en la Meseta, lo que ha conducido a distintos intentos de explicación de este singular fenómeno. En sí, la pluralidad de vectores que rigen el hecho social de la muerte, como siempre sucede, condiciona la comprensión del problema, propiciando en este caso una imagen arqueológica algo confusa y de gran variabilidad en sus manifestaciones, al margen de los mecanismos y principios ideológicos que le dieron sentido. Se trata de identificar y definir de modo preciso el resultado de tales comportamientos, esto es, el registro arqueológico que nos llega, pero también, con mayor trascendencia, aquellas claves generales que regulan la función funeraria más allá de su aspecto final. La representatividad de la población que allí se encuentra, la propia naturaleza de los espacios con evidencias esqueléticas, cómo funciona la dinámica de formación del relleno, si realmente puede hablarse o no de sepulturas, si constituyen una expresión de la norma o, por el contrario, no son más que testigos de la excepción... son, entre otras, cuestiones que aún requieren de una precisa definición arqueológica.

Para terminar, si se pretende avanzar de forma sustancial en el conocimiento del mundo de la muerte en Cogotas I, ello pasa indefectiblemente por la aplicación de criterios de caracterización y explicación que atiendan a la totalidad de acciones y resultados que conforman un depósito con restos humanos, tal y como propugna la Tanatoarqueología (Duday et alii, 2009). A tal efecto, se requiere una lectura integradora de los procesos antedeposicionales, deposicionales y postdeposicionales que actúan en estos contextos hasta el presente: número de individuos, sexo y grupos de edad representados, tratamientos aplicados al cadáver antes de su sepultura, cómo y dónde se produjo el proceso de descomposición, cuándo puede hablarse de depósitos en dos o más tiempos, en qué estado llegan los cuerpos o despojos y qué procesos tafonómicos acontecen ya sea en un enclave de procedencia previo como en su emplazamiento final, cuáles de esos factores están asociados a los gestos culturales vinculados a la manipulación o transporte de los restos humanos y cuáles no, etc. Aunque algunas de estas variables — como las relativas a la estructura demográfica (sexo y edad) e incluso marcadores de esfuerzo, morbilidad, etc - son contempladas cada vez más en los trabajos sobre Cogotas I, es mucho menos usual el análisis de los agentes postdeposicionales que durante o tras la conformación del depósito han afectado a las manifestaciones bioantropológicas. Y sin embargo, el entendimiento y explicación de nuestra realidad arqueológica exige el máximo esfuerzo para caracterizar los comportamientos culturales frente a otros posibles procesos que inciden sobre los restos humanos. Solo de este modo estaremos en condiciones de afrontar las costumbres mortuorias de estas gentes y de distinguir las estrictamente funerarias y otras prácticas de más amplio alcance pero también ancladas en una compleja ideología de la muerte. 


\section{Agradecimientos}

Agradecemos al Ministerio de Economía y Competitividad el soporte financiero de la presente investigación (proyectos HAR2009-10105 y HAR2013-41851-P, del Plan Nacional I+D+i); a la Junta de Castilla y León, el apoyo de nuestro trabajo museístico, y especialmente a $\mathrm{D}^{\mathrm{a}}$ María Mariné Isidro, directora del Museo de Ávila; al Prof. Tomasz Goslar (Poznan Radiocarbon Laboratory) por las dataciones AMS; y a nuestros amigos los Dres. Jose Francisco Fabián García (Arqueólogo Territorial de Ávila) y Antonio Blanco González (Universidad de Salamanca) y la empresa Strato Arqueología (Valladolid), que muy generosamente nos han suministrado información y fotografías de la excavación en el enterramiento del Cerro de la Cabeza.

\section{BIBLIOGRAFÍA}

Alacet Arqueólogos (2008): Informe técnico sobre los trabajos de control arqueológico de la obra de construcción de la "Autovía A-66. Tramo Cazada de ValduncielSalamanca" (Salamanca). Inédito. Servicio Territorial de Cultura de la Junta de Castilla y León. Salamanca.

Aliaga Almela, Raquel, Megías González, Marta (2011): Los Berrocales (Madrid): un yacimiento de la Edad del Bronce en la confluencia Manzanares-Jarama. Universidad Autónoma, Madrid.

Almagro-Gorbea, Martín (1986): "Bronce Final y Edad del Hierro. La formación de las etnias y culturas prerromanas”. En F. Jordá Cerdá, M. Pellicer Catalán, P. Acosta Martínez y M. Almagro-Gorbea, Prehistoria. Madrid: Gredos. Historia de España, I, pp. 340-532.

Álvarez Sanchís, Jesús Rafael (1999): Los Vettones. Madrid: Real Academia de la Historia.

Arnáiz Alonso, Miguel Ángel, Montero Gutiérrez, Juan (2003-04): “El yacimiento de Cogotas I 'Tres Chopos-Abarre' (Villegas, Burgos): esbozo paleoeconómico e implicaciones en las formas de organización social”. Boletín del Seminario de Estudios de Arte y Arqueología, LXIX-LXX, pp. 75-114.

Barroso Bermejo, Rosa, Camino Mayor, Jorge, Bueno Ramirez, Primitiva y Balbin Behrmann, Rodrigo de (2007): Fuentenegroso. Un enterramiento del I milenio a.C. en la sierra de Cuera, Asturias. Oviedo : KRK ediciones.

Barroso, Rosa, Bueno, Primitiva, González, Armando, Candelas, Nieves, Rojas, Juan Manuel y López Sáez, Jose Antonio (2014): “Enterramientos de la Edad del 
Bronce en la Meseta Sur peninsular a partir del Sector 22, Yuncos (Toledo)”. Munibe. Antropologia-Arkeologia, 65, pp. 117-136.

Bello, Silvia y Andrews, Peter (2006): “The intrinsic pattern of preservation of human skeletal and its influence on interpretation of funerary behaviors”. En R. Gowland y C. Knüsel (eds.), Social archaeology of funerary remains. Oxford: Oxbow Books, pp. 1-13.

Binford, Lewis R. (1981): Bones. Ancient men and modern myths. New York-LondonSan Diego: Academic Press.

Blanco-González, Antonio (2010): El poblamiento del bronce final y primer hierro en el sector meridional de la submeseta norte. Tesis doctoral. Universidad de Salamanca. Accesible en http://gredos.usal.es/xmlui/handle/10366/76407 (consultado el $10 / 11 / 2017$ ).

Blasco, Ma Concepción y Rovira, Salvador (1992-3): “La metalurgia del cobre y del bronce en la región de Madrid”. Tabona, VIII (2), pp. 397-415.

Blasco, M M Concepción, Sánchez-Capilla, $M^{\mathrm{a}}$ Luisa y Calle, Juana (1991): "Enterramientos del horizonte Protocogotas en el valle del Manzanares". Cuadernos de Prehistoria y Arqueología Universidad Autónoma de Madrid, 18, pp. 55-112.

Blasco, Concepción, Blanco, Juan Francisco, Liesau, Corina, Carrión, Elena, García, Jesús, Baena, Javier, Quero, Salvador y Rodríguez de la Esperanza, M ${ }^{\mathrm{a}}$ Jesús (2007): El Bronce Medio y Final en la región de Madrid. El poblado de la Fábrica de Ladrillos (Getafe, Madrid). Madrid: Universidad Autónoma de Madrid. Estudios de Prehistoria y Arqueología Madrileñas 14-15.

Botella, Miguel C., Alemán, Inmaculada y Jiménez, Sylvia A. (1999): Los huesos humanos. Manipulación y alteraciones. Barcelona: Ediciones Bellaterra.

Boulestin, Bruno (2016): “Norme funéraire: illusions et verités”. En M. Lauwers y A. Zemour (dirs.), Qu'est-ce ce qu'une sepulture? Humanités et systèmes funéraires de la Préhistoire à nos jours. Antibes: Editions APDCA, pp. 363-377.

Bronk Ramsey, Christopher (2017): “Methods for Summarizing Radiocarbon Datasets”. Radiocarbon, 59 (6), pp. 1809-1833

Camarós, Edgar, Cueto, Marián, Teira, Luis C., Tapia, Jesús, Cubas, Miriam, Blasco, Ruth, Rosell, Jordi, y Rivals, Florent (2013): "Large carnivores as taphonomic agents of space modification: an experimental approach with archaeological implications”. Journal of Archaeological Science, 40(2), pp. 1361-1368. 
Celis Sánchez, Jesús, Delibes de Castro, Germán, Fernández-Manzano, Julio y Grau Lobo, Luis (eds.) (2007a): El hallazgo leonés de Valdevimbre y los depósitos del Bronce Final Atlántico en la Península Ibérica. Valladolid-León: Junta de Castilla y León-Diputación de León.

Celis Sánchez, Jesús, Delibes de Castro, Germán, Fernández-Manzano, Julio, Grau Lobo, Luis, Hernández Valverde, Miryam V., Herrán Martínez, José Ignacio y Rovira Llorens, Salvador (2007b): "Consideración final: El depósito de Valdevimbre y la sociedad Cogotas I”. En J. Celis Sánchez, G. Delibes de Castro, J. Fernández-Manzano y L. Grau Lobo (eds.), El hallazgo leonés de Valdevimbre y los depósitos del Bronce Final Atlántico en la Península Ibérica. Valladolid-León: Junta de Castilla y León-Diputación de León, pp. 168-175.

Colard, Thomas, Delannoy, Yang, Naji, Stephan, Rottier, Stéphane y Blondiaux, Joel (2014): "The utilisation of carnivore scavenging evidence in the interpretation of a protohistoric French pit burial”. Journal of Archaeological Science, 52, pp. 108115 .

Delibes de Castro, Germán (1978): "Una inhumación triple de facies Cogotas I en San Román de Hornija (Valladolid)”. Trabajos de Prehistoria, 35, pp. 225-250.

Delibes de Castro, Germán y Val Recio, Jesús M. del (2005-2006): “Espiraliformes de plata de la cueva de la Vaquera (Segovia): un probable conjunto votivo de los inicios de la Edad de Bronce”. Munibe Antropologia - Arkeologia, 57, pp. 301313.

Delibes de Castro, Germán, Fernández-Manzano, Julio y Herrán Martínez, José Ignacio (2007): “Los Bronces de Valdevimbre y la metalurgia Cogotas I”. En En J. Celis Sánchez, G. Delibes de Castro, J. Fernández-Manzano y L. Grau Lobo (eds.), El hallazgo leonés de Valdevimbre y los depósitos del Bronce Final Atlántico en la Península Ibérica. Valladolid-León: Junta de Castilla y León-Diputación de León, pp. 106-131.

Duday, Henry, Cipriani, Anna M., y Pearce, John (2009): The Archaeology of the Dead. Lectures in Archaeothanatology. Oxford: Oxbow Books.

Esparza Arroyo, Ángel, Velasco Vázquez, Javier y Delibes de Castro, Germán (2012a): "HUM 2005-00139: Planteamiento y primeros resultados de un proyecto de investigación sobre la muerte en Cogotas I”. En Rodríguez Marcos y FernándezManzano, (2012), pp. 259-320.

Esparza Arroyo, Ángel, Velasco Vázquez, Javier y Delibes de Castro, Germán (2012b): "Exposición de cadáveres en el yacimiento de Tordillos (Aldeaseca de la Frontera, Salamanca). Perspectiva bioarqueológica y posibles implicaciones para el estudio del ritual funerario de Cogotas I”. Zephyrus, 69, pp. 95-128. 
Esparza, Ángel; Palomo-Díez, Sara; Velasco-Vázquez, Javier; Delibes, Germán, Arroyo, Eduardo y Salazar-García, Domingo C. (2017): "Familiar Kinship? Palaeogenetic and isotopic evidence from a triple burial of the Cogotas I archaeological culture (Bronze Age, Iberian Peninsula)". Oxford Journal of Archaeology, 36 (3), pp. 223-242.

Etxeberria, Francisco (1994): “Aspectos macroscópicos del hueso sometido al fuego. Revisión de las cremaciones descritas en el País Vasco desde la Arqueología”. Munibe Antropologia-Arkeologia, 46, pp. 111-116.

Fabián, José F., Strato y Blanco-González, Antonio (2010): “El enterramiento en fosa del Cerro de la Cabeza (Ávila). La cuestión funeraria en el Bronce Final / Hierro I en el sur-oeste de la Meseta Norte”. En P. Bueno, A. Gilman, C. Martín y J. Sánchez-Palencia (eds.), Arqueología, Sociedad, Territorio y Paisaje. Homenaje a María Dolores Fernández-Posse. Madrid: CSIC. Bibliotheca Praehistorica Hispana XXVIII, pp. 183-194.

Faith, Tyler, Marean, Curtis W., y Behrensmeyer, Anna K. (2007): “Carnivore competition, bone destruction, and bone density”. Journal of Archaeological Science, 34, pp. 2025-2034.

Fernández Castro, María Cruz (1997): La prehistoria de la Península Ibérica. Barcelona: Crítica.

Fernández Manzano, Julio y Herrán Martínez, José I. (2012): “La metalurgia de Cogotas I”. En Rodríguez Marcos y Fernández-Manzano (2012), pp. 39-58.

Fernández Manzano, Julio, Herrán Martínez, José I. y Rovira Llorens, Salvador (2005): "Los depósitos metálicos burgaleses y la metalurgia del Bronce Final en la Meseta Norte: Algunas Reflexiones". Boletín del Seminario de Estudios de Arte y Arqueología, 71, pp. 137-159.

Fernández-Posse, Ma Dolores. (1998): La investigación protohistórica en la Meseta y Galicia. Madrid: Editorial Síntesis.

Fosse, Philip, Selva, Nuria, Wajrak, Adam, Fourvel, Jean B., Madelaine, Stéphane, ... y Haynes, Gary. (2012): "Bone modification by modern wolf (canis lupus): a taphonomy study from their natural feeding places”. Journal of Taphonomy 10(3), 197-217.

Haglund, William (1997): "Dogs and coyotes: Postmortem involvement with human remains”. En W. Haglund y M. Sorg (eds.), Forensic taphonomy. The postmortem fate of human remains. Boca Raton: CRC Press, pp. 367-381..

Haynes, Gary (1980): "Evidence of carnivore gnawing on Pleistocene and recent mammalian bones”. Paleobiology, 6(3), pp. 341-351. 
Haynes, Gary (1983): "A guide for differentiating mammalian carnivore taxa responsible for gnaw damage to herbivore limb bones”. Paleobiology, 9(2), pp. 164-172.

Herrán Martínez, José I. (2008): Arqueometalurgia de la Edad del Bronce en Castilla y León. Valladolid: Universidad de Valladolid-Junta de Castilla y León. Studia Archaeologica, 95.

Insoll, Timothy (2015): “Constructing Ancestors in Sub-Saharan Africa”. En C. Renfrew, M. J. Boyd y I. Morley (eds.), Death Rituals, Social Order and the Archaeology of Immortality in the Ancient World: 'Death Shall Have No Dominion'. Cambridge: Cambridge University Press, pp. 153-167.

Kjorlien, Yvonne, Beattie, Owen y Peterson, Arthur (2009): "Scavenging activity can produce predictable patterns in surface skeletal remains scattering: Observations and comments from two experiments”. Forensic Science International, 188, pp. 103-106.

Madgwick, Richard (2008): "Patterns in the modification of animal and human bones in Iron Age Wessex: Revisiting the excarnation debate”. En O.P. Davis, N.M. Sharples y K.E.. Waddington (eds.), Changing Perspectives on the First Millennium BC. Oxford: Oxbow, pp. 99-118.

Madgwick, Richard y Mulville, Jacqueline (2015): "Reconstructing depositional histories through bone taphonomy: extending the potential of faunal data”. Journal of Archaeological Science, 53, pp. 255-263.

McKinley, Jacqueline (2000): “The analysis of cremated bone”. En M. Cox y S. Mays (eds.), Human osteology in archaeology and forensic science. GMM: Londres, pp. 403-421.

Moraitis, Konstantinos y Spiliopoulou, Chara (2010): "Forensic implications of carnivore scavenging on human remains recovered from outdoor locations in Greece”. Journal of Forensic and Legal Medicine, 17, pp. 298-303.

Palomino, Ángel Luis, Negredo, M ${ }^{\mathrm{a}}$ José y Abarquero, Francisco Javier (1999): "Cabañas, basureros, silos y tumbas en el yacimiento de El Cerro, La Horra (Burgos): a vueltas sobre el significado de un campo de hoyos en la Edad del Bronce en la Meseta”. Numantia, 7, pp. 21-41.

Parkinson, Jennifer A., Plummer, Thomas W. y Bose, Rebecca (2014): “A GIS-based approach to documenting large canid damage to bones". Palaeogeography, Palaeoclimatology, Palaeoecology, 409, pp. 57-71. 
Pérez Villa, Alberto (2015): Pautas funerarias de la Edad del Bronce en la cuenca media y alta del Tajo. Madrid: CSIC. Bibliotheca Praehistorica Hispana, 31.

Rodríguez-Marcos, José Antonio y Fernández-Manzano, Julio (eds.) (2012): Cogotas I: Una cultura de la Edad del Bronce en la Península Ibérica. Valladolid: Universidad de Valladolid.

Rovira Llorens, Salvador, Delibes de Castro, Germán, Fernández-Manzano, Julio y Herrán Martínez, José Ignacio (2004): “Composición de coladas y tratamientos postfundición en la metalurgia calcolítica y de la Edad del Bronce de la Submeseta Norte Española: Estudio de una trayectoria tecnológica”. Kobie, 6, pp. 231-247.

Sala, Nohemí, Arsuaga, Juan Luis y Haynes, Gary (2014): “Taphonomic comparison of bone modifications caused by wild and captive wolves (Canis lupus)”. Quaternary International, 330, pp. 126-135.

Scott, David A. (1991): Metallography and microstructure of ancient and historic metals. Los Angeles: The Getty Conservation Institute.

Smith, Martin (2006): "Bones chewed by canids as evidence for human excarnation: a British case study”. Antiquity, 80, pp. 671-685.

Steadman, Dawnie y Worne, Heather (2007): “Canine scavenging of human remains in an indoor setting”. Forensic Science International, 173(1), pp. 78-82.

Thomas, Louis-V. (1982): La Mort africaine: idéologie funéraire en Afrique Noire. Paris: Payot,

Trujillo-Mederos, A., Bosch, P., Pijoan, C. y Mansilla, J. (2015): "Savoury Recipes and the Colour of the Tlatelcomila Human Bones”. Archaeometry, 58, pp. 688-704.

Velasco-Vázquez, Javier y Esparza-Arroyo, Ángel (2016): "Muertes ritualizadas en la Edad del Bronce de la Península Ibérica: un enterramiento inusual en Los Rompizales (Quintanadueñas, Burgos)”. Munibe Antropologia - Arkeologia, 67, pp. 75-106.

Walker, Phillip, Miller, Kevin y Richman, Rebecca (2008): "Time, temperature, and oxygen availability: an experimental study of the effects of environmental conditions on the color and organic content of cremated bone". En E. Schmidt y S. Symes (eds.), The Analysis of Burned Human Remains. San Diego: Academic Press, pp, 129-135. 